\title{
Grenze, Handel, Heiligtum - die Bedeutung ethnisch übergreifender Kultstätten Südkampaniens in archaischer Zeit
}

Das Sarnotal wird zwar vom Golf von Salerno mit seinem gebirgigen Hinterland und der daran anschließenden Küstenebene des Flusses Sele durch die Sorrentinische Halbinsel mit den Monti Lattari topographisch deutlich getrennt, dennoch bildeten diese Landschaften seit der frühen Eisenzeit einen zusammenhängenden Kulturraum mit gemeinsamer historischer Entwick-

Der Dank des Autors gilt den Verantwortlichen des Fonds zur Förderung der österreichischen Wissenschaft (FWF), die durch die Finanzierung des Forschungsprojektes „Etrusker, Griechen und Italiker in Südkampanien“ die Erstellung der vorliegenden Arbeit ermöglicht haben, der wissenschaftlichen Leiterin dieses Forschungsprojektes, Fr.

Prof. L. Aigner-Foresti, sowie Prof. G. Dobesch für wertvolle Hinweise und Anregungen.

Abkürzungen mehrfach zitierter Werke:

Atti Magna Grecia, 1992: Atti e Memorie della Società Magna Grecia, Omaggio a P. Zancani Montuoro, N.S. III,1 (1992).

Briquel, 1984: Les Pèlasges en Italie. Recherches sur l'histoire de la légende, Rom 1984 (Bibl. des Écoles Françaises d'Athenes et de Rome 252).

Cerchiai, 1987: L. Cerchiai, Il processo di strutturazione del politico: i Campani, AION 9 (1987) 41-54.

ders., 1995: I Campani, Mailand 1995.

Culti della Campania: I Culti della Campania antica, Atti del conv. int. di studi in ricordo di N. Valenza Mele (Neapel 1995) Rom 1998.

Eschebach, Pompeji: H. u. L. Eschebach, Pompeji vom 7. Jh. v. Chr. bis 79 n. Chr., Köln 1995.

Fiammenghi, 1985: C. A. Fiammenghi, Agropoli. Primi saggi di scavo nell'area del Castello, AION 7 (1985) 53-74.

Fratte, 1990: Un insediamento etrusco-campano, Hgg. G. Greco/A. Pontrandolfo, Modena 1990.

Greco, 1979: E. Greco, Ricerche sulla chora poseidoniate: il „paesaggio agrario” dalla fondazione della città alla fine del sec. IV a. C., Dial. Arch. N.S. 1 (1979) 7-26.

Greco, 1992: G. Greco, La ripresa delle indagini allo Heraion di Foce Sele, Atti Magna Grecia, 1992, 249-258.

dies., 1999: Santuari extraurbani tra periferia cittadina e periferia indigena, in: La colonisation greque en Méditerranée occidental, Actes de la rencontre en hommage à G. Vallet (Rom - Neapel 1995) Rom 1999 (Coll. de l'École Française de Rome 251) 231-247.

Heraion I, II: P. Zancani Montuoro/U. Zanotti Bianco, Heraion alla Foce del Sele, Bd I, Rom 1951; Bd. II, Rom 1954.

Horsnaes, 1991: H. W. Horsnaes, The Ager Picentinus, Acta Hyperborea 3 (1991) 219234.

Magna Grecia, 1994: Magna Grecia. Etruschi. Fenici, Atti del XXXIII. Conv. di studi sulla Magna Grecia 1993, Tarent 1994.

Mertens, 1993: D. Mertens, Der alte Heratempel in Paestum und die archaische Baukunst in Unteritalien, Mainz 1993.

Paestum, 1987: G. Avagliano/M. Cipriani, Paestum, Città e territorio nelle colonie greche d'occidente I, Tarent 1987.

Poseidonia-Paestum: Poseidonia-Paestum, Atti del XXVII. Conv. di studi sulla Magna Grecia 1987, Tarent 1988. 


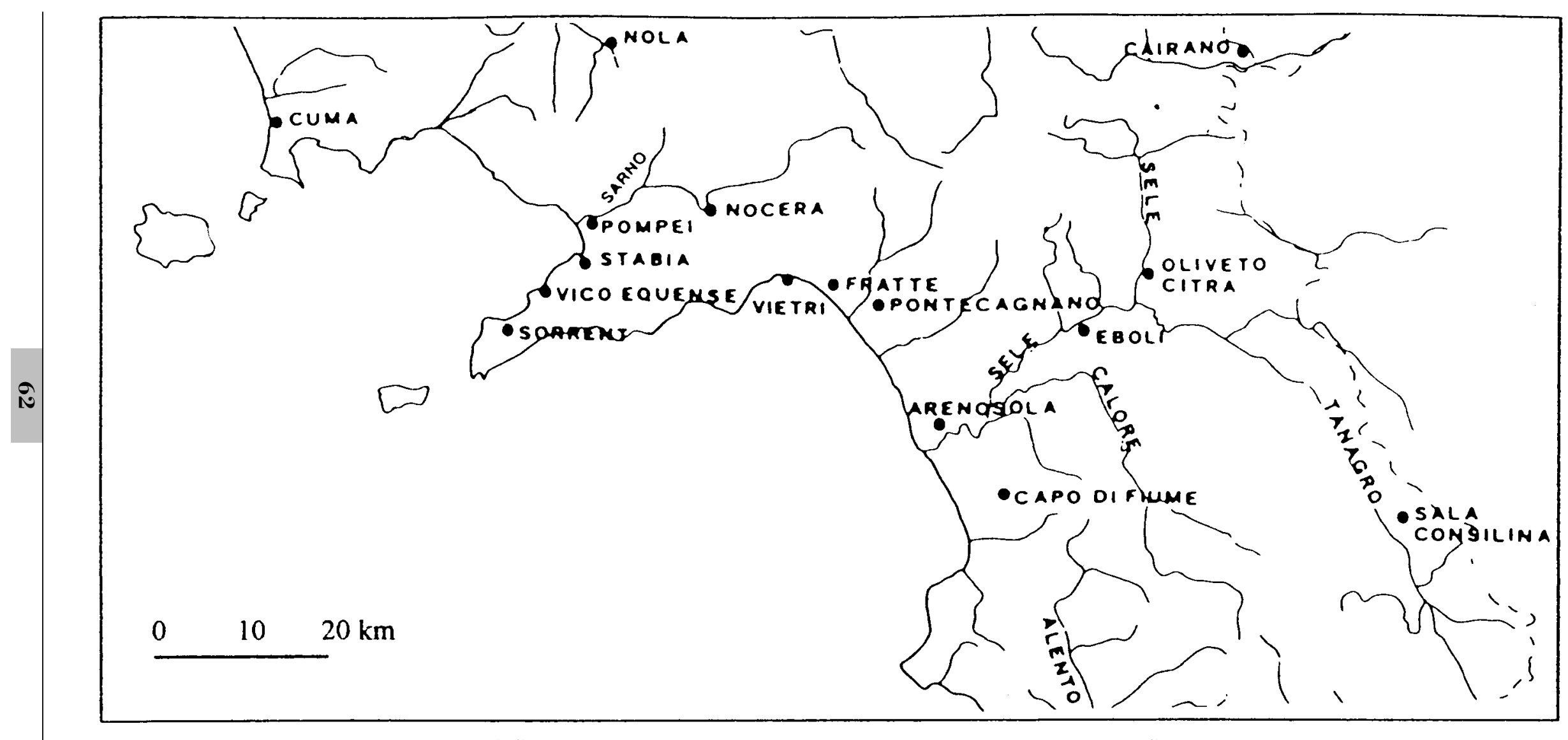

Karte 1: Südkampanien mit den im Text genannten italischen und etruskischen Siedlungen 
lung (Karte 1$)^{1}$ - in diesem Sinn gehören sie auch heute wieder einer politischen Region, Campania, an. Seit der Gründung der griechischen Kolonie Poseidonia im späten 7. Jh. ${ }^{2}$ lebten im südlichsten Teil dieses Kulturraums, in der Küstenebene des Sele, Angehörige der etruskischen, italischen und griechischen Volksgruppe unmittelbar nebeneinander; diese traten darüber hinaus in wechselseitigen Kontakt mit den Bewohnern der Siedlungen im Bereich des heutigen Salerno und im Sarnotal ${ }^{3}$.

Ziel der vorliegenden Arbeit ist es, die auf verschiedenen Ebenen faßbare politische Bedeutung der an Territorialgrenzen sowie Handelsplätzen eingerichteten Kultstätten Südkampaniens zu verdeutlichen. Eine entsprechende Frage wurde bisher nur in allgemeiner Form gestellt ${ }^{4}$ und bezüglich Kampaniens nur im Rahmen allgemeiner Untersuchungen des Zusammenlebens der unterschiedlichen Volksgruppen ${ }^{5}$ bzw. unter spezifischen Gesichtspunkten einzelner Heiligtümer behandelt ${ }^{6}$. Hier sollen nun die gut dokumentierten to-

1 Seit dem 9. Jh. v. Chr. lassen sich hier zwei vor allem aufgrund ihrer Bestattungssitten gut unterscheidbare Kulturen nachweisen: die Fossakultur, deren Angehörige ihre Verstorbenen in Gruben (fossae) beerdigten, sowie eine ihre Toten verbrennende eisenzeitliche Kultur die der zeitgleichen Villanova-Kultur in Südetrurien nahe steht. Trotz regional unterschiedlicher Ausformungen dieser großen Kulturgruppen im Golf von Neapel (Fossakultur des Küstenbereichs und Villanovakultur von Capua) und am Golf von Salerno bzw. in dessen Hinterland (Fossakultur von Oliveto Citra-Cairano und Villanovakultur von Pontecagnano) standen die Bewohner beider Landschaften stets in engem Kontakt; der Golf von Neapel und das Sarnotal sind also historisch nicht vom Golf von Salerno und seinem Hinterland zu trennen. In diesem Sinn behandelten zuletzt sowohl B. d'Agostino, Le genti della Campania antica, in: Italia omnium terrarum alumna, Hg. G. Pugliese Carratelli, Mailand 1988 (Antica madre) 531-89, als auch Cerchiai, 1995, im Rahmen ihrer umfassenden Darstellungen der Geschichte Kampaniens beide Landschaften. Zu den genannten eisenzeitlichen Kulturen und ihren wechselseitigen Beziehungen B. d'Agostino, 1988, 531-537; Cerchiai, 1995, 9-11.

2 Alle Jahresangaben sind v. Chr. Von den archäologischen Belegen des Zeitpunktes der Gründung von Poseidonia wird gleich noch ausführlicher zu sprechen sein.

3 Strab. 5,4,13 (C 251) gibt die Entfernung der Wegstrecke von Pompeji über Nuceria nach Markina, der ersten Siedlung am Golf von Salerno, mit 120 Stadien an. Zur Landverbindung vom Sarnotal an den Golf von Salerno T. Cinquantaquattro, Dinamiche insediative nell'agro picentino dalla protostoria all'età ellenistica, AION 14 (1992) 245 und Karte auf S. 246. Die Identifizierung von Markina ist umstritten. B. d.'Agostino, Marcina? DialArch 2 (1968) 139-151, schlug eine Gleichsetzung mit den heutigen Vietri sul mare vor, was jedoch von E. Greco in einem Diskussionsbeitrag in AION 6 (1984) 266, sowie zuletzt auch von Cerchiai, 1995, 124, in Frage gestellt wurde. Cerchiai spricht sich dagegen vorsichtig für eine Identifizierung mit der in Fratte, einem Vorort des heutigen Salerno, gefundenen Siedlung aus. In diesem Sinn auch L. Vecchio, Le fonti storiche, in: Fratte, 1990, 19 f., der das Forschungsproblem umreißt und die der Diskussion zugrunde liegenden Quellen anführt.

4 P. G. Guzzo, Schema per la categoria interpretativa del „santuario di frontiera“, Scienze dell'Ant. 1 (1987) 373-379.

5 Zuletzt etwa B. d'Agostino, La Campania e gli Etruschi, in: Magna Grecia 1994, 431-448; F. Zevi, I Greci, gli Etruschi, il Sele, in: I Culti della Campani antica, 3-27; im Rahmen ganz Großgriechenland einschließender Überlegungen G. Greco, 1999.

${ }^{6}$ So jüngst L. Cerchiai, Aspetti della funzione politica di Apollo in area tirrenica, in: Culti della Campania antica, 123-128, zu Art und Bedeutung des Apollonkultes in Pompeji. I. Krauskopf, Il ciclo delle Metope del primo Thesauros, Atti Magna Grecia, 1992, 219-231 und E. Simon, Hera ed Eracle alla foce del Sele e nell'Italia centrale, Atti Magna Grecia, 1992, 207-219, haben sich mit den Beziehungen Poseidonias zu den Etruskern befaßt, wie sie sich aufgrund des Bildprogramms des als ,erster Thesauros' bezeichneten Kultbaus im Heraion am Sele erschließen lassen; zu diesem Bildprogramm zuletzt auch umfassend C. Masseria/M. Torelli, Il mito all'alba di una colonia greca. Il programma figurativo delle metope dell'Heraion alla Foce del Sele, in: Le mythe grec dans l'Italie antique. Fonction et 
pographischen Gegebenheiten und die reichen Bodenfunde in Poseidonia, Pontecagnano und Pompeji, aber auch jene im Sarnotal, am Golf von Salerno sowie im Hinterland von Poseidonia herangezogen und gemeinsam untersucht werden, um exemplarisch die politische Bedeutung ethnisch übergreifender Kultstätten am Schnittpunkt dreier unterschiedlicher Kulturen darzustellen?

\section{Landnahme und Abgrenzung des Territoriums: die Gründung von Poseidonia}

Strabon unterscheidet bei seiner Beschreibung der Gründung von Poseidonia, 6,1,1 (C 252), eine direkt am Meer gelegene und durch eine künstliche Befestigungsanlage ( $\varepsilon \varepsilon \hat{\chi} \chi 0 \varsigma)$ geschützten Ansiedlung von einer Ansiedlung in der

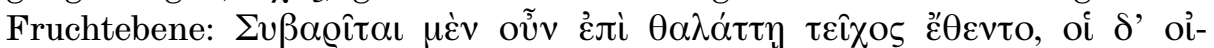

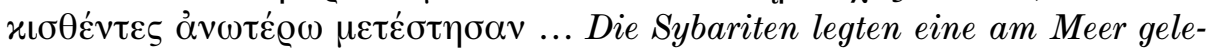
gene befestigte Siedlung an, die Gründer der Stadt aber ließen sich weiter nördlich nieder.... ${ }^{8}$. Archäologische und topographische Untersuchungen haben weitere Erkenntnisse über die Siedlungen in der Fruchtebene sowie am Kap Agropoli erbracht und zu einer erneuten kritischen Diskussion der Lokalisierung des $\tau \varepsilon i ̂ \chi 0 \varsigma$ geführt ${ }^{9}$. Jüngst durchgeführte Grabungen haben auch bestätigt, daß das Heraheiligtum im Mündungsbereich des Sele bereits an der Wende vom 7. zum 6. Jh. gegründet wurde ${ }^{10}$.

image, Hg. F.-H. Massa-Pairault, Rom 1999 (Coll. Ec. Fr. Rome 253) 205-262. Rein baugeschichtlich bleibt die anregende Arbeit von D. Mertens, Elementi di origine etrusco-campana nell'architettura della Magna Grecia, in: Magna Grecia, 1994, 207-212.

7 Eine vergleichbare Untersuchung würde sich auch für den westsizilischen Raum anbieten, wo ebenfalls drei unterschiedliche Volksgruppen, nämlich Punier, Sikaner (bzw. Elymer) und Griechen direkt aufeinander trafen. Einzelne Aspekte dieses Zusammenlebens wurden von M. Giangiuglio, Greci e non-greci in Sicilia alla luce dei culti e delle leggende di Eracle, in: Forme di contatto e processi di trasformazione nelle società antiche, Atti del congr. di Cortona 1981, Pisa/Rom 1983 (Coll. de l'Ecole Française de Rome 67) 785-846, aufgrund des Herakleskultes behandelt.

8 Die Deutung der Schlüsselbegriffe $\tau \varepsilon \hat{\chi} \chi 0 \varsigma$ als eine erste befestigte Ansiedlung di-

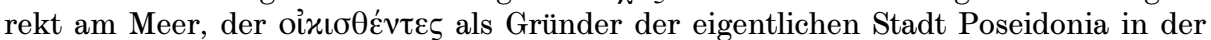
Fruchtebene und von $\alpha$ v $\omega \tau \varepsilon \dot{\varrho} \omega$ als Angabe der Lage der Stadt bezüglich der ursprünglichen Ansiedlung am Meer folgt den Ergebnissen der ausführlichen Textanalyse durch M. Guarducci, Alcune monete di Posidonia e la fondazione dell'antica città, in: Gli archeologi italiani in onore di A. Maiuri, Cava dei Tirreni 1965, 203-217; M. Mello, Strabone V,4,13 e le origini di Poseidonia, PP 117 (1967) 401-424; E. Greco, Il TEIXO $\Sigma$ dei Sibariti e le origini di Posidonia, Dial. d'Arch. 8 (1974/5) 104-115; ders., 1979; G. Pugliese Carratelli, Per la storia di Posidonia, in: Poseidonia-Paestum, $13 \mathrm{ff}$., sowie dem darauf bezogenen Diskussionsbeitrag von A. Mele, S. 618.

9 Aufgrund der Grabungsergebnisse am Kap Agropoli, die unten noch ausführlicher zu diskutieren sein werden (vgl. bes. Anm. 55 u. 60), sollte diese künstliche Befestigungsanlage der Sybariten nicht am ohnehin natürlich geschützten Kap Agropoli, sondern im Bereich der Selemündung oder im Küstenabschnitt nahe dem eigentlichen Stadtgebiet gesucht werden; in diesem Sinn schon E. Greco, 1974/5, s. o. Anm. 8, sowie zuletzt vor allem H. Tréziny, Autour de la fondation de Poseidonia, AION XIV (1992) 4561. Die Befunde im späteren Stadtgebiet von Poseidonia werden gleich vorgelegt und diskutiert.

10 J. de la Genière/G. Greco, Beuaucoup de questions et quelques résponses au santuaire de Héra à Foce del Sele, in: I Culti della Campani antica, Atti del Conv. Int. di Studi in recordo di N. Valenza Mele, Neapel 1995, Rom 1998, 37-43, bes. 39. Von den Befunden im Heraion am Sele wird nun bezüglich der Bedeutung dieses Heiligtums als Kultstätte an der Grenze des poseidoniatischen Territoriums ausführlicher zu sprechen sein (vgl. bes. die in Anm. 36 genannten Arbeiten). 


\section{Die Inbesitznahme der Fruchtebene}

An zentraler Stelle der Fruchtebene südlich des Sele besetzten griechische Siedler im späten 7. Jh. ein damals weniger als einen Kilometer von der Küste entfernt gelegenes Travertinplateau, das sich einige Meter über das Meeresniveau erhob $^{11}$. Dabei verzichteten sie zwar auf eine nach fortifikatorischen oder verkehrstechnischen Gesichtspunkten besonders günstige Lage - sie siedelten weder direkt am Meer, an einer Flußmündung oder in unmittelbarer Nähe sonstiger natürlicher Verkehrswege, noch wählten sie eine durch besonderen natürlichen Schutz ausgezeichnete Stelle - ließen sich aber dafür inmitten eines landwirtschaftlich vielfältig nutzbaren Gebietes nieder (Karte 2$)^{12}$. Dieses war allerdings am Ende des 7. Jh.s bereits lange von Italikern bewohnt ${ }^{13}$. Die archäologischen Nachweise dieser italischen Präsenz im Bereich der späteren Stadt Poseidonia enden an der Wende vom 7. zum 6. Jh. und zeigen, daß die Italiker die Griechen nicht entscheidend an ihrer Landnahme hindern konnten ${ }^{14}$.

Vielmehr zeigt ein bereits im frühen 6 . Jh. in Fonte, etwa $14 \mathrm{~km}$ nordöstlich von Poseidonia und nahe dem heutigen Albanella gegründetes Heiligtum der Göttin Hera (Karte 2) ${ }^{15}$, daß zu diesem Zeitpunkt die gesamte Fruchtebene von den Griechen kontrolliert wurde. In Fonte betont ein schmales Flußtal die natürliche Grenze zwischen der Fruchtebene und dem hügelig-gebirgigen Hinterland. Diese topographischen Gegebenheiten sprechen dafür, daß das hier gegründete Heraion zunächst primär die Grenze zwischen der von den Griechen beanspruchten Fruchtebene und dem italischen Hinterland markieren sollte. Etwa zeitgleich mit der Einrichtung dieses Heiligtums enden die Bestattungen in einer italischen Nekropole, die nur etwa einen Kilometer östlich von Fonte, auf dem Hügel von Tempalta, gefunden wurde. Den selben Befund zeigt eine etwas weiter nordwestlich, im heutigen Rovine di Palma nachweisbare italischen Ansiedlung ${ }^{16}$. Auch hier enden die Belege einer italischen Anwesenheit im frühen 6. Jh. Dieser parallele Befund macht es

11 Unmittelbar vor der Porta della Marina steigt das Niveau heute rasch von 5 auf $12 \mathrm{~m}$ über dem Meer an. Ein weiterer Anstieg auf 19,3 m, die höchste Erhebung in weitem Umkreis, erfolgt unmittelbar vor der Porta della Giustizia. Eine Karte mit detaillierter Angabe der Höhenlinien ist in Paestum, 1987, Tav. 11, publiziert. Zum Küstenverlauf in antiker Zeit und der damit verbundenen Frage nach dem Hafen Poseidonias vor allem G. Schmiedt, Antichi porti d'Italia. Parte seconda: i porti delle colonie greche, L'Universo 46,2 (1966) 311 ff., bes. Karte auf S. 312; M. Guy, La costa, la laguna e l'insediamento di Poseidonia-Paestum, in: Paestum. La città e il territorio, Enciclopedia multimediale Rom 1990 (Quaderno di documentazione) 66f. mit Fig. 1, u. 75. Zu den topographischen Gegebenheiten weiters J. Delezir-M. Guy und E. Greco in ihren Beiträgen in PoseidoniaPaestum, 463-470 bzw. 471-499, bes. 475ff.

12 E. Greco, 1979, $22 \mathrm{f}$. Während in der Ebene nördlich und östlich des Stadtgebietes vor allem Ackerbau getrieben wurde, dürfte das hügelige Gelände am Rand der Fruchtebene mit ihrem Wiesen- und Waldbestand vor allem zur Viehzucht und Holzwirtschaft genützt worden sein.

13 Dies bezeugen Streufunde aus dem Stadtgebiet selbst und Bestattungen in Gaudo, etwa 1,5 km nördlich sowie in Capodifiume, etwa 4,5 km nordöstlich von Poseidonia (diese letztgenannte Siedlung ist hier auf Karte $1 \mathrm{im}$ Anhang verzeichnet). Zu allen Siedlungen E. Greco, in: D. Theodorescu/E. Greco, Poseidonia-Paestum II, L'agora, Rom 1983 (Coll. de l'École Française de Rome 42) 73f.; ders., in: Poseidonia-Paestum, 474 f.; zur Nekropole von Capodifiume schon G. Voza, Necropoli di Capodifiume, in: Mostra della preistoria e protostoria nel Salernitano, Neapel 1962, $79 \mathrm{f}$.

14 E. Greco, 1983, s. o. Anm. 13.

15 Paestum, 1987, 30f. Nr. 33 (Fonte); Poseidonia-Paestum, 428-430, Taf. LXX (Abbildungen hier gefundener Votivstatuetten des 6. Jh.).

16 Paestum, 1987, 27f. Nr. 20. 


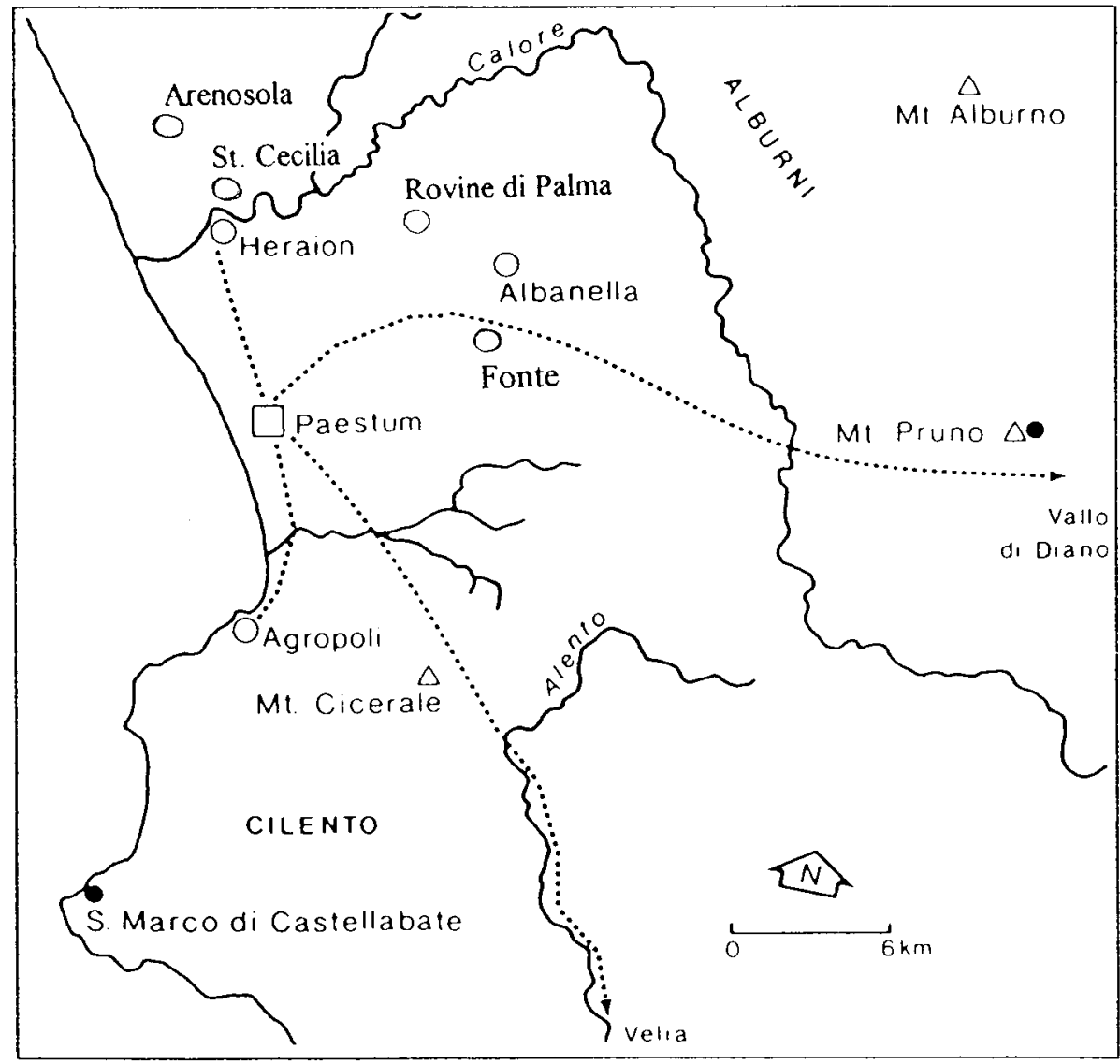

Karte 2: Das Territorium von Poseidonia (Paestum) mit seinem Hinterland

wahrscheinlich, dal3 der Bruch in der Siedlungskontinuität mit der Ankunft der Griechen zu verbinden ist. Ein friedliches Nebeneinander von Griechen und Italikern ist in Fonte dagegen erst um Mitte des 6. Jh. durch Grabfunde zu belegen: zu diesem Zeitpunkt bestatteten Angehörige der italischen Volksgruppe ihre Verstorbenen nach eigenem Ritus in unmittelbarer Nähe des griechischen Heiligtums ${ }^{17}$. Diesem friedlichen Zusammenleben von Italikern und Griechen entspricht die ab der zweiten Hälfte des 6. Jh. nachweisbare Nutzung des Tals von Fonte als Teil einer Handelsroute über den Monte Pruno weiter in das Vallo di Diano ${ }^{18}$.

\section{Zwischenergebnis}

Die Ankunft der Griechen und ihre Landnahme in der fruchtbaren Küstenebene führte mit hoher Wahrscheinlichkeit zur Verdrängung eines Großteils jener Italiker, deren Lebensgrundlage der Ackerbau gewesen war.

17 Paestum, 1987, 30f. Nr. 33 (Fonte): Kolonettenkratere aus Bucchero „pesante“ kampanischer Machart, Schalen, Becken und Weinamphoren aus Bucchero sowie „ionische" Kylikes.

18 Der Verlauf dieses Handelsweges südlich des Flusses ist durch Funde in Capodimonte und Tempalta, fünf bzw. 14 km nordöstlich des späteren Poseidonia, zu verfolgen und hier auf Karte 2 im Anhang verzeichnet; dazu (bezüglich der Funde in Tempalta) Paestum, $29 \mathrm{f}$. Nr. 31. Er führte in östlicher Richtung weiter durch die Küstenebene, am Monte Pruno vorbei und schließlich ins Hinterland bis nach Sala Consilina. Damit folgte er in etwa dem Verlauf der modernen Nationalstraße Nr. 166. Zu einer am Monte Pruno nachweisbaren italischen Siedlung noch ausführlicher unten mit Anm. 132. 
Einen Hinweis darauf, daß es im Zuge der griechischen Inbesitznahme der Fruchtebene auch zu bewaffneten Auseinandersetzungen zwischen Griechen und Italikern kam, geben vielleicht die nur in der Frühphase der griechischen Anwesenheit nachweisbare Verehrung der Hera als aktive Kämpferin, als Promachos ${ }^{19}$, sowie jene Schwerter, Lanzen und Pfeile, die in allen poseidoniatischen Heiligtümern Heras gefunden wurden ${ }^{20}$. Allerdings wurden Waffen nicht nur aus Anlaß militärischer Erfolge gestiftet ${ }^{21}$, sondern auch von Privatleuten der Hera Hoplosmia geweiht ${ }^{22}$. Da Weihinschriften, die eine gestiftete Waffe eindeutig als Beutestück kennzeichnen würden, in Poseidonia bisher nicht nachweisbar sind ${ }^{23}$, und es auch keine sonstigen Hinweise auf Kämpfe zwischen Griechen und Italikern gibt, können derartige bewaffnete Auseinandersetzungen daher derzeit nur vermutet werden.

Spätestens zu Beginn des 6. Jh., dem Zeitpunkt der Einrichtung des Heraheiligtums in Fonte, war die griechische Kontrolle der Fruchtebene jeden-

19 So zuletzt I. Solima, Era, Artemide e Afrodite in Magna Grecia e Grecia. Dee armate o dee belliche? MEFRA 110 (1998) 387 f., Fig. 1. Abbildung einer Hera-PromachosStatuette auch in Poseidonia-Paestum, Taf. LVI (oben links, allerdings mit falscher Beischrift). Demselben Typus entspricht vielleicht auch eine im nördlichen Stadtheiligtum gefundene weibliche Statuette, die G. Avagliano in Poseidonia-Paestum, 376, erwähnt.

${ }_{20}$ Eine detaillierte Untersuchung und zusammenhängende Publikation dieses Materials steht bislang aus. Voranzeigen der Waffenfunde im Heraion und in sonstigen Heiligtümern durch G. Greco, Heraion di Foce Sele. Nuove prospettive di ricerca, in: Momenti di storia salernitana nell'antichità, Atti del Conv. Naz. AICC di Salerno-Fisciano 1988, Hg. I. Gallo, 49 ff., bes. 54 u. dies., 1992. Zu den Funden im südlichen Stadtheiligtum weiters M. Cipriani, in: Poseidonia-Paestum, 380 mit Taf. LV.

21 Allgemein zur Sitte von Waffenweihungen durch die Griechen W. K. Pritchett, The Greek State at War III. Religion, Berkeley u. a. 1979, 240 ff.; A. H. Jackson, Hoplites and the Gods: The dedication of captured armour, in: Hoplites: The classical greek battle experience, Hg. V. D. Hanson, 1991, 228-249.

22 Vor allem die im Heraion am Sele gefundenen Miniaturwaffen sind als derartige Stiftungen an Hera Hopolosmia aufzufassen; zu diesen und weiteren Waffenfunden G. Greco, 1988, s.o. Anm. 20, 54; zum Aspekt der Hera Hoplosmia vor allem die unten Anm. 41 genannten Arbeiten.

${ }^{23}$ In Sinn einer Anrufung Heras als Schützerin der poseidoniatischen Krieger wurde die Aufschrift auf einer im südlichen Stadtheiligtum gefundenen, unregelmäßig geformten

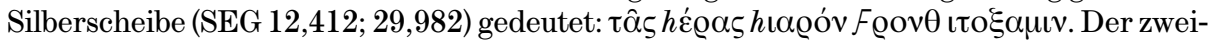
te Teil dieser Inschrift ist höchst umstritten und wurde von M. Guarducci, Dedica arcaica

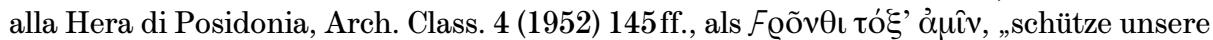
Bogen“, aufgelöst. L. H. Jeffery, Further comments on archaic greek inscriptions, ABSA 50 (1955) 78, las dagegen fronth (ph?) itoxamin und deutete dies als ,Italic or prae-Italic 'im Sinn von „Besitz der Hera“ oder „X weihte mich“; vgl. auch dies., in: The local scripts of archaic Greece, Oxford ${ }^{2} 1990,252,260$ Nr. 3. Linguistische Überlegungen bei R. Arena, Iscrizioni greche arcaiche di Sicilia e Magna Grecia IV. Iscrizioni delle colonie achee, Alessandria 1996, 45 Nr. 19, Taf. VI,1, der sich vorsichtig für die Interpretation M. Guarduccis ausspricht. Zuletzt las A. La Regina, Dono degli oligarchi di Amina all'Heraion di Posido-

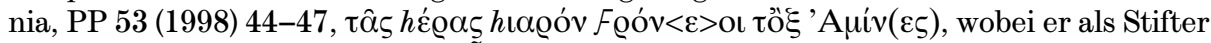
dieses Weihgeschenk an Hera die ท๊๊̣óvol (entsprechend @óvıoı) in Sinn der Angehörigen der Oberschicht von Amina (das La Regina am ehesten mit der in Fratte bei Salerno gefundenen Siedlung gleichsetzen will) identifiziert. Auch diese Deutung muß allerdings aufgrund der unsicheren Lesung der Inschrift hypothetisch bleiben. Plausibel ist weiterhin eine Verbindung der in großen Schriftzeichen an zentraler Stelle der Silberscheibe eingetieften Buchstabengruppe $\alpha \mu \nu$ mit der Aufschrift AMI auf jenen inkusen Silbermünzen, die im 6. Jh. von einer sowohl wirtschaftlich wie kulturell unter dem Einfluß von Sybaris stehenden politischen Gemeinschaft ausgegeben wurden. Zu diesen Münzen N. F. Parise,

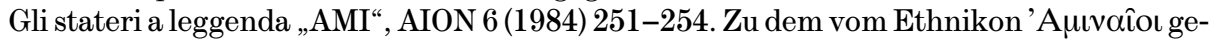
bildeten Ortsnamen Amina bzw. Aminaia G. Bailo Modesti, Lo scavo nell'abitato antico di Pontecagnano e la coppa con l'iscrizione AMINA[-] AION 6 (1984) 237 ff.; L. Cerchiai, I Campani, Mailand 1995, 77. 110f. und zuletzt La Regina, PP 53 (1998) 45-47. 
falls weitgehend unumstritten. Um die Mitte des 6 . Jh. war hier auch jenes friedliche Zusammenleben von Griechen und Italikern möglich, das die Voraussetzung einer in der zweiten Hälfte des 6 . Jh. nachweisbaren handelstechnischen Nutzung der Landverbindung von Poseidonia ins Vallo di Diano und in weiterer Folge zu den achäischen Städten Unteritaliens, vor allem Sybaris und Metapont, schuf ${ }^{24}$. Es erscheint plausibel, den Bedeutungsgewinn dieser Landverbindung als Folge des Konfliktes der achäischen Städte Unteritaliens mit Lokroi und dessen Verbündeten Rhegion zu sehen, der erstmals bei der Zerstörung von Siris faßbar ist und wenig später in der Schlacht an der Sagra erneut offen zum Ausbruch kam ${ }^{25}$ - auf dem Landweg konnten achäische Kaufleute jedenfalls die Fahrt durch die von Rhegion kontrollierte Meerenge von Messina vermeiden, wenn sie ihre Waren in das italischen Hinterland, aber auch nach Poseidonia bzw. zu dessen etruskischen Nachbarn bringen wollten ${ }^{26}$.

\section{Das Mündungsgebiet des Sele}

Die Küstenebene nördlich des Sele wurde von der etruskischen Siedlung Pontecagnano aus kontrolliert, die schon an der Wende vom 9. zum 8. Jh. wenig östlich des Flusses Picento angelegt worden war ${ }^{27}$. Die hier ansässigen Etrusker dehnten ihren Einfluß allmählich nach Süden bis zum Sele aus. Dies zeigt sich in den Grabbeigaben aus jener Nekropole, die um das Jahr 700 in Arenosola, kaum vier Kilometer nördlich des Sele, angelegt wurde (Karte 2) ${ }^{28}$ : die meisten der hier gefundenen keramischen Beigaben waren in Pontecagnano hergestellt worden ${ }^{29}$. Es ist also wahrscheinlich, daß der Sele bereits zum Zeitpunkt der Ankunft der Griechen die politische Grenze zwischen dem etruskischen ager Picentinus im Norden und der italisch besiedelten Küstenebene südlich des Flusses markierte, eine Funktion, die auch in der viel jüngeren literarischen Überlieferung beschrieben ist ${ }^{30}$.

$24 \mathrm{Zu}$ dieser schon oben Anm. 18.

25 Siris hatte der Überlieferung zufolge von Lokroi Epizephyrioi Hilfe gegen die achäischen Aggressoren Sybaris, Metapont und Kroton erhalten. Schon wenig später kam es zwischen Lokroi und Kroton zu einer Schlacht am Fluß Sagra, wobei nach Strab. 6,1,10 (C261) die Rheginer auf seiten von Lokroi gegen die Achäer standen. Allerdings ist der Zeitpunkt der entsprechenden kriegerischen Auseinandersetzungen unklar, da die bei Strabon, Justin 20, 2.1-3.9 und Paus. 3, 19.11-12 überlieferten Berichte über die Schlacht an der Sagra stark von propagandistischen Tendenzen geprägt sind, wie M. Giangiuglio, Le tradizioni leggendarie intorno alla battaglia della Sagra, MEFRA 95 (1983) 473-521, deutlich gezeigt hat. Zu Zeitpunkt und Umständen der Zerstörung von Siris und zur Schlacht an der Sagra G. L. Huxley, in: Siris e l'influenza ionica in occidente, Atti del XX. Conv. di studi sulla Magna Grecia 1980, Tarent 1981, 34-38; vgl. auch A. J. Graham, in: CAH III, $3^{2}$, 1982, 182. 193f., sowie P. J. Bicknell, The date of the battle of the Sagra river, Phoenix 20 (1966) 294-301.

${ }_{26} \mathrm{Zu}$ den Handelsinteressen von Sybaris und deren wahrscheinlicher Bedeutung im Rahmen der Gründung von Poseidonia ausführlicher unten Anm. 53.

27 Dazu ausführlich Cerchiai, 1995, 51-68; Cerchiai verweist 228f. auch auf grundlegende Publikationen der Ergebnisse der Grabungen in den Nekropolen und im Wohnbereich dieser Siedlung.

28 Publiziert von A. Marzullo, La necropoli dell'Arenosola a destra della Foce del Sele, Rassegna Storica Salernitana 2,1 (1938) 3-26, und B. d.'Agostino, Arenosola, in: Mostra della Preistoria e Protostoria nel Salernitano, Neapel 1962, $90 \mathrm{ff}$. Zusammenfassung der Ergebnisse und deren Interpretation sowie ältere Literatur bei Horsnaes, 1991, bes. $222 \mathrm{ff}$. Die letzten Beisetzungen fanden hier um das Jahr 575 statt.

29 So die Bestattungen in der Nekropole von Capodifiume, 4,5 km nordöstlich des späteren Poseidonia; zu diesen schon oben Anm. 13.

30 Strab. 5,4,13 (C 251), der den Sele als Grenzfluß zwischen dem Gebiet der Pikenter und Lukanien bezeichnet. Dazu E. Greco, 1983, s. o. Anm. 13, 73f., sowie A. Mele, Da Poseidonia a Paestum, in: Paestum, Hg. F. Zevi, Neapel 1990, 23-34. 
Gleichzeitig bezeugt der Fund einzelner etruskischer Schmuck- und Gebrauchsgegenstände (vor allem Gefäße pontecagnanischer Machart) in italischen Gräbern südlich des Flusses die Handelsbeziehungen der Angehörigen der etruskischen und italischen Volksgruppe zu beiden Seiten des Sele ${ }^{31}$. Eine entscheidende Rolle bei diesen Handelskontakten spielte jene Stelle an der ersten Flußbiegung, wo in der Antike eine Furt das Durchqueren des Sele ermöglichte (Karte 2). Hier, etwa fünf Kilometer flußaufwärts, an einer etwas erhöhten Stelle mit dem modernen Flurnamen St. Cecilia, weisen reiche keramische Oberflächenfunde eine Frequentation von der späten Bronzezeit bis in römische Zeit nach, ohne daß sich vor einer systematischen archäologischen Untersuchung des Geländes differenziertere Aussagen machen ließen. Eine antike Straße läßt sich allerdings von St. Cecilia bis zur Nekropole von Arenosola verfolgen und führte wahrscheinlich weiter nach Pontecagnano ${ }^{32}$. Weitere Streufunde stammen aus einer nördlich des Heraheiligtums nachweisbaren Nekropole, die jedoch ebenfalls noch nicht systematisch untersucht ist ${ }^{33}$. An der Furt bei St. Cecilia endete jedenfalls der für größere Schiffe befahrbare Abschnitt des Sele. Hier mußten also alle Waren, die über das Meer heran geschafft wurden, ausgeladen und auf dem Landweg oder auf kleineren Flußschiffen ins Hinterland weiter transportiert werden, wobei zwei Handelsrouten zur Wahl standen. Zum einen jene durch das Tal des Sele nach Eboli und Oliveto Citra, dann dem Tanagro folgend nach Buccino und schließlich nach Sala Consilina im Vallo di Diano ${ }^{34}$, zum anderen jene durch die Küstenebene südlich des Sele und das daran anschließende Bergland ebenfalls nach Sala Consilina ${ }^{35}$.

Bereits im späten 7. richteten achäische Griechen auf dem St. Cecilia gegenüber liegenden südlichen Flußufer ein Heiligtum der Göttin Hera ein und statteten dieses mit einem Altar und wohl noch im ersten Viertel des 6. Jh. mit einer Säulenhalle aus ${ }^{36}$. Zusammen mit den ältesten Weihegaben griechischer Machart wurden hier aber auch Gefäße gefunden, die von Töpfern in Pontecagnano in etruskisch-italischer Tradition hergestellt worden waren ${ }^{37}$.

$31 \mathrm{Zu}$ den Befunden oben Anm. 13.

32 D. Gasparri, La fotointerpretazione archeologica nella ricerca storico-topografica sui territori di Pontecagnano, Paestum e Velia, AION 11 (1989) 262. Die Nekropole der Siedlung in St. Cecilia befand sich wohl nicht nördlich des Heraion, wie noch die Autoren von Heraion I, 22, meinten, sondern eher in Arenosola, wofür sich zuletzt Horsnaes, 1991, $228 \mathrm{f}$., aussprach.

33 Beide Fundstellen wurden bereits von den Ausgräber des Heraion angezeigt, U. Zanotti-Bianco, Le scoperte allo Heroion del Sele, Le vie d'Italia 46 (1940) 895; Heraion I, 22; zu diesen auch G. Greco, in: Poseidonia-Paestum, 389; Horsnaes, 1991, 228f. mit Anm. 4.

${ }^{34} \mathrm{Zu}$ den engen Beziehungen zwischen Pontecagnano, Eboli und Buccino M. Cipriani, Eboli preromana. I dati archeologici: analisi e proposte di lettura, in: Italici in Magna Grecia. Lingua, insediamenti e strutture, Hg. M. Tagliente, Atti del Conv. di Acquasparta 1986, Venosa 1990, 119-145.

35 Zum Verlauf dieses Handelsweges schon oben Anm. 18.

${ }^{36}$ Heraion I, 25 ff., fig. 5; G. Tocco Sciarelli/J. de la Genière/G. Greco, in: Poseidonia-Paestum, 385 ff.; G. Tocco Sciarelli, Heraion di Foce Sele. Nuove Prospettive di ricerca, in: Momenti di storia salernitana nell'antichità, Atti del Conv. Naz. AICC di SalernoFisciano 1988, Hg. I. Gallo, 35-41, bes. 38. Zur Funktion der erwähnten Säulenhalle G. Kuhn, Untersuchungen zur Funktion der Säulenhalle in archaischer und klassischer Zeit, JdI 100 (1985) $264 \mathrm{ff}$.

37 Heraion I, 22. Eine Publikation des reichen Votivmaterials aus dem Heraion wird von einer Arbeitsgemeinschaft unter Leitung von G. Greco und M. Dewailly vorbereitet; Poseidonia-Paestum, 394; G. Greco, 1988, s. o. Anm. 20. Siehe vor allem auch 
Der daraus erschließbare friedliche Kontakt der Griechen mit ihren Nachbarn am anderen Flußufer entspricht dem äußeren Erscheinungsbild des Heiligtums als einem allgemein zugänglichen Areal, das auch nicht durch eine Temenosmauer abgeschlossen wurde ${ }^{38}$. Dies macht es wahrscheinlich, daß zumindest ein Teil der in Pontecagnano bzw. in dessen Umland hergestellten Gefäße im frühen 6. Jh. im Zuge religiös motivierter Besuche des Heiligtums von Etruskern gestiftet wurden ${ }^{39}$. Ein friedliches Zusammenleben am Sele ist auch aus dem Befund der oben schon genannten Nekropole in Arenosola erschließbar. Dieser Friedhof wurde ohne Unterbrechung bis gegen 575, also noch während der ersten beiden Generationen griechischer Anwesenheit, genützt ${ }^{40}$.

Das unmittelbar an der Grenze des griechischen zum etruskischen Territorium eingerichtete Heiligtum der Hera diente also wohl auch und vor allem der Definition und Bestätigung der durch den Fluß Sele gegebenen natürlichen politischen Grenze durch die Griechen. Gleichzeitig zeigten sie damit aber auch ihre Bereitschaft zu einem friedlichen Zusammenleben mit ihren etruskischen Nachbarn, wobei der vielfältige Charakter der in Poseidonia verehrten Hera ${ }^{41}$ einer Deutung nach dem Verständnis etruskischer Besucher des Heiligtums entgegenkam ${ }^{42}$. In diesem Sinn wurde im Heraion am Sele im dritten Viertel des 6. Jh. ein Kultbau, der sogenannte „erste Thesauros“43, mit figuralen Metopen verziert, deren mythologische Motive sich sämtlich auch in der etruskischen Kunst finden ${ }^{44}$. Am zahlreichsten sind dabei Taten des Herakles, dessen eigenständige Verehrung durch die Etrusker gut bezeugt ist ${ }^{45}$. Gleichzeitig dehnte Herakles den Kreis der durch die Metopendarstellungen angesprochenen Personen auch auf die Italiker aus - schließlich

Greco, 1992, 254, Taf. LVI,1, mit der Publikation einer im Heraion gefundenen, aber in Pontecagnano hergestellten Schale mit einer Verzierung „à chevrons fluttuanti“.

38 Das Areal des Heraheiligtums am Sele wurde dagegen wohl nur durch lagunenartige Nebenarme des Flusses begrenzt, wie G. Tocco Sciarelli, J. de la Genière u. G. Greco, in: Poseidonia-Paestum, 386 (vgl. auch G. Tocco Sciarelli, 1988, s. o. Anm. 36, 38) vermuteten. Eine als Schutz gegen etwaige bewaffnete Überfälle gedachte Temenosmauer wie sie etwa bei Strab. 11,8,4 für ein in Kappadokien der Göttin Anaïtis errichtetes Heiligtum beschreibt, gab es im Heraion am Sele jedenfalls nicht.

${ }^{39}$ So schon die Ausgräber P. Zancani Montuoro und U. Zanotti-Bianco in Heraion I, 22.

$40 \mathrm{Zu}$ den Befunden oben Anm. 28.

41 A. M. Ardovino, Culti di Paestum antica e del suo territorio, Salerno 1986, $113 \mathrm{ff}$; zuletzt auch M. Cipriani, Il ruolo di Hera nel santuario meridionale di Poseidonia, in: Héra. Images, espaces, cultes, Actes du Coll. int. du centre de rech. arch. de l univ. de Lille III e de l'ass. P.R.A.C., Hg. J. de La Genière, Neapel 1997 (Coll. du Centre J. Bérard 15) 211-225.

42 Dabei ist an das Heiligtum jener Gottheit in Pyrgi, dem Hafen von Caere, zu erinnern, die von den Etruskern als Uni, von den Karthagern als Astarte und von den Griechen als Hera verehrt wurde. Zu diesem Heiligtum zusammenfassend G. Colonna, Il santuario di Leucotea-Ilizia a Pyrgi, in: Santuari d'Etruria, Katalog zur Ausstellung in Arezzo 1985, Hg. G. Colonna, Florenz 1985, 127 ff., bes. 134; M. Cristofani, s. v. Pirgi, BTCG XIII, Pisa-Rom 1994, 579-590.

43 Heraion I, 25-32 bzw. Heraion II (baulicher Befund); jetzt vor allem K. Junker, Der ältere Tempel im Heraion am Sele. Verzierte Metopen im architektonischen Kontext, Köln u. a. 1993, $44 \mathrm{f}$. $58 \mathrm{f}$, der den Kultbau überzeugend als Tempel deutet und auch die hier genannte Datierung aufgrund stilistischer Argumente vertritt.

${ }^{44}$ I. Krauskopf, Il ciclo delle Metope del primo Thesauros, Atti Magna Grecia, 1992, 219-231.

45 E. Simon, Hera ed Eracle alla foce del Sele e nell'Italia centrale, Atti Magna Grecia, 1992, 207-219; vgl. zuletzt auch Greco, 1999, 235. 
wurde Herkle im 6. Jh. von Latium bis Kampanien in mehreren Orten verehrt, in denen Italiker und Etrusker zusammentrafen ${ }^{46}$. Bezüglich seiner Bekanntheit in Südkampanien ist vor allem auf die Verehrung dieses Helden in und um Pompeji hinzuweisen ${ }^{47}$, da, wie unten noch zu zeigen sein wird, Beziehungen Poseidonias zu dieser kampanischen Siedlung im späten 6. Jh. aufgrund formaler Übereinstimmungen der Verzierung öffentlicher Großbauten nachweisbar sind.

Einem friedlichen Kontakt der unterschiedlichen Volksgruppen im Heraheiligtums am Sele entspricht schließlich auch die bei Strab. 6,1,1 (C 252) überlieferte Tradition einer Gründung dieses Kultortes durch Jason auf der Argonautenfahrt. Im Zuge der in den italischen Westen verlegten Version dieser Fahrt pflegte der Held friedlichen Umgang mit der ansässigen Bevölkerung $^{48}$. Der Mythos einer Gründung durch die Argonauten konstruierte also nachträglich ein in mythische Vorzeit zurückreichendes friedliches Zusammentreffen von Etruskern, Italikern und Griechen an der Mündung des Sele ${ }^{49}$.

\section{Zwischenergebnis}

Die Ansiedlung der Griechen an der lukanischen Küste war von unterschiedlichen Motiven und Faktoren bestimmt, wobei die Landnahme in der Küstenebene wohl primär der Bereitstellung ausreichend fruchtbaren Ackerlandes für alle Neusiedler diente; dies dürfte gleichzeitig zur Verdrängung zumindest eines Teils der bereits ansässigen italische Bevölkerung geführt haben. Die Einrichtung eines Heraheiligtums in Fonte, am Ostrand der Fruchtebene, ist daher am ehesten als äußeres Zeichen der dauerhaften griechischen Kontrolle des Hinterlandes zu sehen. Ein solches Zeichen wurde wohl notwendig, da die Fruchtebene während des gesamten 6. Jh. von den Griechen zwar landwirtschaftlich genutzt wurde, die poseidoniatischen Bauern jedoch in erster Linie innerhalb bzw. in unmittelbarer Umgebung des eigentlichen Stadtgebietes wohnten $^{50}$. Allmählich übernahm dieses Heiligtum wohl auch die

46 Ausführlich zusammengestellt von M. Torelli, Gli aromi e il sale. Afrodite ed Eracle nell'emporia arcaica dell'Italia, in: Ercole in occidente, Hg. A. Mastrocinque, Trento 1993, 91-117.

47 Zur literarischen Tradition der Anwesenheit des Herakles in Pompeji zuletzt L. Cerchiai, Aspetti della funzione politica di Apollo in area tirrenica, in: Culti della Campania antica, 125; zu den Kultorten des Herkules in Pompeji D. Camardo/A. Ferrara, Petra Herculis: un luogo di culto alle foci del Sarno, AION 12 (1990) 169-175; vgl. auch M. Torelli, s. o. Anm. 46, $115 \mathrm{f}$. Zu einer möglichen Verehrung des Helden im archaischen Tempel am Forum Triangulare Eschebach, Pompeji, 36 mit Anm. 110.

48 Apollon. Rhod. 4, $659 \mathrm{ff}$.

49 In diesem Sinn schon A. Mele, Diskussionsbeitrag in: Poseidonia-Paestum, 620, dann ausführlicher L. Breglia Pulci Doria in: Mito e storia in Magna Grecia, Atti del XXXVI. Conv. di studi sulla Magna Grecia 1996, Tarent 1997, 242ff. bes. 245; vgl. zuletzt auch G. Greco, 1999, $235 \mathrm{f}$.

50 Zur Besiedlungsgeschichte des poseidoniatischen Territoriums ausführlich Greco, 1979; vgl. auch die von J. Pedley, 1990, s. o. Anm. 18, 98 Abb. 60, publizierte Karte der griechischen Bestattungsplätze des 6 bis 4 . Jh. Erst ab etwa der Mitte des 4 . Jh. sind mehrere Siedlungen und zahlreichere Gehöfte im Hinterland nachweisbar, wobei diese auch und vor allem von den italischen Bewohnern des poseidoniatischen Territoriums bewohnt und bewirtschaftet wurden, wie jüngst D. Asheri, Processi di „decolonizzazione“ in Magna Grecia: il caso di Poseidonia lucana, in: La colonisation greque en Méditerranée occidental, Actes de la rencontre en hommage à G. Vallet (Rom-Neapel 1995) Rom 1999 (Coll. de l'École Française de Rome 251) 366 f., betonte. 
Funktion eines Ortes der friedlichen Begegnung von Griechen und Italikern, wie sie das Heraion am Sele an der Nordgrenze des poseidoniatischen Territoriums von Anfang an erfüllt haben dürfte ${ }^{51}$.

Mit der Gründung des Heiligtums der Hera an der Selemündung konnten die Griechen die durch den Fluß vorgegebene politische Grenze zum etruskischen Territorium bestätigen und die Art der Beziehungen zu diesen Nachbarn definieren ${ }^{52}$. Die Gründung des Heraion als ein auch für die etruskischen und italischen Nachbarn offener Kultort sollte in diesem Sinn wohl die von den Griechen angestrebten friedlichen Kontakte zum Ausdruck bringen. Dabei dürften von Anfang an handelspolitische Überlegungen eine wichtige Rolle gespielt haben, da sich die Griechen in unmittelbarer Nähe einer Furt festsetzten, wo der Verkehr auf und über den Sele kontrolliert werden konnte. Es erscheint daher plausibel, die Gründung des Heraion als handelspolitische Maßnahme von Sybaris zu sehen, das hier einen Ort zum Warenaustausch mit den Etruskern gesucht hätte ${ }^{53}$. Dies findet eine Stütze in der bereits eingangs zitierten knappen Beschreibung der Gründung von Poseidonia bei Strabo 6,1,1 (C 252), im Zuge deren er die Errichtung einer direkt am Meer ge-

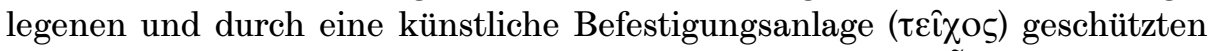

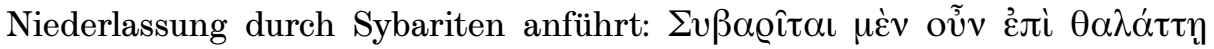
$\tau \varepsilon i ̂ \chi 0 \varsigma ~ \varepsilon ้ \theta \varepsilon v \tau \mathrm{o}^{54}$. Von einer anderen, am Kap Agropoli an der Südgrenz des poseidoniatischen Territoriums eingerichteten Handelsstation, und der dort nachweisbaren engen Verbindung von Handels- und Kultaktivitäten, wird nun die Rede sein; unter diesem Gesichtspunkt sollen in weiterer Folge auch die Befunde des Heraion am Sele sowie jene amderer Heiligtümer Südkampaniens untersucht werden.

\section{HaNdel und HeIligtum}

\section{Die Siedlung am Kap Agropoli}

Folgt man der lukanischen Küste von Süden nach Norden so bildet das Kap Agropoli die letzte bedeutende Erhebung vor der Küstenebene des Sele $(\text { Karte } 2)^{55}$. Die beiden Buchten südlich und nördlich dieses Kaps boten natürlich geschützte Landeplätze auf dem Seeweg nach Norden ${ }^{56}$. In der nördlichen

${ }^{51}$ In diesem Sinn schon G. Tocco Sciarelli, in: Poseidonia-Paestum, $445 \mathrm{f}$.

${ }^{52}$ In diesem Sinn zuletzt auch Greco, 1999, 235.

53 Daß Sybaris in Handelskontakt mit den Etruskern stand, ist aus Tim. FGrH 566 F 50, Z. 3 f., erschließbar, wo die Etrusker (Tv@@ๆvoí) als Freunde der Sybariten bezeichnet und bezüglich ihrer engen Beziehungen zu den Sybariten den Milesiern gleichgestellt werden. Da eine Nutzung des Handelsweg über Land, durch das Vallo di Diano nach Poseidonia, erst ab etwa der Mitte des 6. Jh. v. Chr. nachweisbar ist (vgl. oben Anm. 18) erscheint es plausibel, daß die Sybariten vorher, d. h. an der Wende vom 7. zum 6. Jh. ihre Handelsinteressen dennoch primär zur See betrieben und mit den Niederlassungen an der lukanischen Küste (Agropoli, Selemündung) Stützpunkte für ihre Handelsaktivitäten mit Kampanien und Etrurien schaffen wollten. Zur Außenpolitik von Sybaris zuletzt umfassend E. Greco, L'impero di Sibari. Bilancio archeologico-topografico, in: Sibari e la Sibaritide, Atti del XXXII. Conv. di Studi sulla Magna Grecia 1992, Tarent 1993, 459-485.

${ }^{54}$ Literaturangaben zu dieser intensiv diskutierten Passage oben Anm. 8.

55 Diese Lokalisierung wurde bereits von P. Zancani-Montuoro, Il Poseidonion di Poseidonia, Archivio Storico per la Calabria e la Lucania XXIII (1954) 165ff., aufgrund topographischer Beobachtungen und auf Basis von Lykophr. Alex. $722 \mathrm{ff}$. vertreten.

56 G. Schmiedt, 1966, s. o. Anm. 11, 314. 
der beiden Buchten mündet darüber hinaus der Fluß Testene ins Meer und erlaubte Seefahrern eine Versorgung mit Trinkwasser. Diese Gegebenheiten kommen der Einrichtung einer Handelsstation auf dem Seeweg vom griechischen Mutterland bzw. von Unteritalien nach Kampanien und weiter nach Etrurien entgegen ${ }^{57}$ und sprechen dafür, daß diese Stelle schon in antiker Zeit als Landeplatz genutzt wurde ${ }^{58}$. Der Fund griechischer Gebrauchskeramik weist darüber hinaus eine kontinuierliche Besiedlung des Hügels von Agropoli seit dem frühen 6. Jh. nach, wobei auch die Verzierung der hier gefundenen Keramik für die Herkunft der Gründer aus Sybaris spricht ${ }^{59}$. Daher ist hier mit hoher Wahrscheinlichkeit eine bereits seit der Wende vom 7. zum 6. Jh. dauerhaft besetzte sybaritische Handelsstation zu lokalisieren ${ }^{60}$.

Griechische Transportamphoren unterschiedlicher Herkunft bezeugen für das ganze 6. Jh. einen regelmäßigen Besuch dieser Siedlung durch Fernhändler zur See und sprechen ebenfalls für ihre Bedeutung als Handelsstation ${ }^{61}$. Die Kontrolle eines derartigen Hafenplatzes war in archaischer Zeit wirtschaftlich überaus lukrativ, wie es Hdt. 1,165,1 bezeugt ${ }^{62}$. In diesem Sinn ist damit zu rechnen, daß die Erhebung von Hafengebühren, die Versorgung von Seefahrern mit Trinkwasser und Lebensmitteln sowie eine Tätigkeit als Zwischenhändler ${ }^{63}$, die wichtigsten Einnahmequellen jener aus Sybaris stammenden Griechen waren, die sich im späten 7. Jh. dauerhaft am Kap Agropoli niederließen.

Diese griechischen Bewohner gründeten wohl auch jenes Heiligtums, das hier bereits seit dem späten 7. Jh. bestand, in dem etwa ein Jahrhundert später ein monumentaler Kultbau errichtet wurde und in dem eine Kultaktivität bis ins 4 . Jh. nachweisbar ist ${ }^{64}$. Die bereits genannten keramischen Funde aus diesem Heiligtum entsprechen in ihrer Art Weihegaben griechischer Händler in Gravisca, dem Hafenplatz von Tarquinia in Etrurien ${ }^{65}$. Dies macht es plau-

57 Zum Verlauf der entsprechenden Seehandelsrouten F. di Bello, Elea-Velia. Polis, zecca e monete di bronzo, Neapel 1997, 46 f. mit Fig. 18.

${ }^{58}$ In diesem Sinn schon E. Greco, 1974/5, s. o. Anm. 8, 104 ff.; vgl. auch ders., $1979,51 \mathrm{ff}$.

${ }_{59}$ Für die achäische Herkunft der hier ansässigen Griechen sprechen Trinkschalen mit einer Randverzierung „a filetti“ wie sie in Sybaris hergestellt wurden und nicht nur am Kap Agropoli, sondern auch in den ältesten Gräbern der Stadt Poseidonia gefunden wurden; Fiammenghi, 1985, 57 f.; zum Material aus den Nekropolen Greco, 1979, 11 Anm. 24.

60 Ergebnisse der archäologischen Untersuchungen bei Fiammenghi, 1985, 53-74, bes. 57 f.; vgl. auch dies., in: Poseidonia-Paestum, 396-398; sowie Paestum, 1987, 47 Nr. 74.

61 Fiammenghi, 1985, $61 \mathrm{f}$.

62 Diesem zufolge hätten die Chioten sich geweigert, den Phokäern nach deren Flucht aus Kleinasien die nahe Chios gelegene Inselgruppe der Oinussen zu verkaufen, aus Furcht, diese könnten den Handelsverkehr von der eigenen Stadt ablenken. Dazu A. Bresson, Les cités greques et leurs emporia, in: L' Emporion, Hgg. A. Bresson/P. Rouillard, Paris 1993, $169 \mathrm{f}$.

63 C. Ampolo, Greci d'occidente, Etruschi, Cartaginesi: circolazione di beni e di uomini, in: Magna Grecia, 1994, 223-252; ders., Tra empória ed emporía: note sul commercio greco in età arcaica e classica, in: AПOIKIA, Scritti in onore di G. Buchner, AION N. S. 1 (1994) 29-36.

${ }^{64}$ Dieser Befund, insbesondere die im 4. Jh. hergestellten Athenastatuetten, erfordert eine erneute Diskussion der von P. Zancani Montuoro, 1954, s. o. Anm. 55, vertretenen Identifikation dieses Kultortes als Heiligtum des Poseidon Enipeios; dazu schon Fiammenghi, 1985, 65f.

65 Wobei durch Weihinschriften eine Verehrung von Apollo, Hera, Aphrodite und Demeter bezeugt ist. M. Torelli, Il santuario greco di Gravisca, PP 32 (1977) 398-458; F. Boitani, Il santuario di Gravisca, in: Santuari d'Etruria, Katalog zur Austellung in Arezzo 
sibel, auch einen Teil der im Heiligtum von Agropoli deponierten Gefäße als Weihungen von Händlern zu deuten, die damit für eine glückliche Ankunft an diesem Landeplatz und für günstige Geschäftsabschlüsse dankten ${ }^{66}$.

\section{Der Handelsplatz an der Selemündung}

Auf die verkehrstechnische Bedeutung des Mündungsgebietes des Sele und insbesondere der Furt an der ersten Flußschleife, sowie auf den aufgrund der ältesten Bodenfunde im Heraheiligtum erschließbaren friedlichen Kontakt der Griechen mit ihren Nachbarn nördlich des Flusses wurde bereits hingewiesen. Daneben wurde das Heiligtum wohl schon seit dem späten 7. Jh. regelmäßig von griechischen Händlern zur See besucht, die im griechischen Mutterland hergestellte korinthische Feinkeramik an den Sele brachten ${ }^{67}$. Diese Seefahrer nützten wahrscheinlich lagunenartige Verzweigungen am Südufer des Flusses als natürlich geschützte Landeplätze ${ }^{68}$. Um die Mitte des 6. Jh.s wurde schließlich eine Säulenhalle errichtet, die auf ein Areal südlich bzw. südöstlich des Heraheiligtums orientiert war und wohl dessen Nutzung als Lande- und Stapelplatz erleichtern sollte ${ }^{69}$.

\section{Öffentliche Baumaßnahmen des frühen 6. Jh.s in Pontecagnano}

Am südlichen Rand von Pontecagnano wurde im frühen 6. Jh. ein offener Platz angelegt und an seiner Westseite durch eine Säulenhalle begrenzt ${ }^{70}$. Die Form dieses Gebäudes spricht für seinen öffentlichen Charakter und gleichzeitig für die Nutzung des Areals davor als Marktplatz. Zwei im späten 4. Jh. geschlossene Votivdepots wurden am Ostrand dieses Platzes gefunden und bezeugen die Existenz eines Heiligtums, wobei die ältesten Weihegaben im späten 7. Jh. hergestellt worden waren ${ }^{71}$.

Auf vier der hier gefundenen Gefäße ist in Buchstaben des achäischen Alphabets АПО $\Lambda$, also der abgekürzte Name des Gottes Apollo, vermerkt. Das älteste derart beschriftete Gefäß ist ein in der zweiten Hälfte des 6. Jh.s hergestellter Kantharos aus Bucchero ${ }^{72}$. Diese Aufschriften sind entweder Sa-

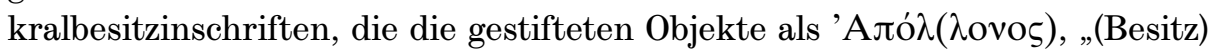

1985, Hg. G. Colonna, Florenz 1985, 141 ff. Zu den jüngsten Grabungsergebnissen F. Boitani/M. Torelli, Un nuovo santuario dell'emporion di Gravisca, in: La colonisation greque en Méditerranée occidental, Actes de la rencontre en hommage à G. Vallet (RomNeapel 1995) Rom 1999 (Coll. de l'École Française de Rome 251) 93-101.

${ }^{66}$ Fiammenghi, 1985, 65f., die sich ebenfalls auf die eben genannten Stiftungen in Gravisca beruft.

67 Eine systematische Publikation des reichen keramischen Fundmaterials aus dem Heraion wird von einer Arbeitsgruppe unter Leitung von G. Greco und M. Dewailly vorbereitet; dazu schon oben Anm. 37.

$68 \mathrm{Zu}$ diesen Gegebenheiten in antiker Zeit M. Guy, 1990, s. o. Anm. 11, 66 f. Fig. 1. Im Bereich südlich des Heiligtums ist wohl, mit G. Schmiedt, 1966, s. o. Anm. 11, 309 f., auch der in römischer Zeit bezeugte Portus Alburnus zu suchen. Anders aber M. Guy, 1990, s. o. Anm. 11, 75.

69 Publiziert von P. Zancani Montuoro, Heraion alla Foce del Sele. Stoa arcaica, Atti e Memorie della Soc. Magna Grecia, N.S. VIII (1967) 7-18; vgl. auch Greco, 1992, 24.

70 Dieser Bereich befindet sich heute zwischen den Straßen Via Verdi und Via Belluno. L. Cerchiai, Nota preliminare sull'area sacra di Via Verdi, in: La ricerca archeologica nell'abitato di Pontecagnano, AION 6 (1984); ders., 1995, 108f.; G. Bailo Modesti, AION 6, 1984, s. o. Anm. 23, 215-245.

71 L. Cerchiai, 1984, s. o. Anm. 70, 248.

72 G. Bailo Modesti, 1984, s. o. Anm. 23, 245 B) Nrn. 1-4; fig. 36 G 2, 3a.b u. 4. 
des (Heiligtums des) Apollo" kennzeichneten, oder Abkürzungen der Weiheformel 'Aлó $\lambda(\lambda \circ v \imath)$ „dem Apollo (zum Geschenk)“73. Einen Hinweis darauf, daß auch aus Südkampanien stammende Italiker dieses Heiligtum des Apollo frequentierten, geben gemeinsam mit diesen griechischen Weihinschriften gefundene Gefäße mit Graffiti eines bäumchenförmigen Buchstabens ${ }^{74}$ jenes oskischen Alphabets, das sonst nur durch Gefäßaufschriften aus Nuceria im Sarnotal sowie aus Vico Equense an der Nordküste der Sorrentinischen Halbinsel bezeugt ist ${ }^{75}$. Der in Pontecagnano verehrte Gott war also den etruskischen und italischen Bewohnern vertraut, konnte aber auch von den achäischen Griechen als Apollo identifiziert und verehrt werden. Dies spricht für die schon oben aufgrund seiner Lage und baulichen Gestaltung als wahrscheinlich aufgezeigten Funktion des Areals vor diesem Heiligtum als ein von Angehörigen aller dreier Volksgruppen genützter Handelsplatz.

Die Anregung zur Wahl Apollos als hier verehrten Gottes kam am ehesten aus dem etruskischen Mutterland, genauer: aus Caere, das im 6. Jh. enge Kontakte zum Apollonheiligtum von Delphi unterhielt und dieses auch als politisch-moralische Instanz anerkannte ${ }^{76}$. In Pyrgi, dem Hafen von Caere, wurde Apollo auch von griechischen Händlern verehrt ${ }^{77}$. Der enge Kontakt Pontecagnanos mit Caere ist durch die Ansiedlung jener caeretaner Meister nachweisbar, die im frühen 6. Jh. in Pontecagnano Werkstätten zur Produktion etruskisch-korinthischer Keramik einrichteten ${ }^{78}$. Weiters ist auf jene gleich noch ausführlicher anzusprechenden Etrusker mit caeretaner Dialekt hinzuweisen, die im 6. Jh. das Apollonheiligtum in Pompeji frequentierten.

\section{РОМРEJ}

Pompeji lag vor dem Ausbruch des Vesuv im Jahr 79 n. Chr. auf einem Höhenrücken, der von einem prähistorischen Lavastrom gebildet worden war. Der Steilabfall dieses Höhenzuges gab die natürlichen Grenzen einer Bebauung im

73 M. L. Lazzarini, Le formule dediche votive nella Grecia arcaica, Rom 1976, $75 \mathrm{ff}$., bes. 78 .

${ }^{74}$ G. Bailo Modesti, 1984, s. o. Anm. 23, 236 mit Anm. 34 u. fig. 33; Cerchiai, 1987, 43.

75 Die Inschriften wurden von G. Colonna, Nuceria Alfaterna, StEtr 42 (1974) 379386 und R. Arena, L'iscrizione di Vico Equense, StEtr 42 (1974) 387-390, publiziert. Zu diesen auch P. Poccetti, Nuovi documenti italici a completamento del manuale di E. Vetter, Pisa 1979, $107 \mathrm{f}$.; R. Wallace, The paleo-oscan Besitzerinschriften from Nocera and Vico Equense, IndogermF 95 (1990) 171-183; M. Cristofani, Sulle più antiche iscrizioni italiche della Campania, in: La presenza etrusca nella Campania meridionale, Atti delle giornate di studio a Salerno e Pontecagnano 1990, Hg. P. Gastaldi/G. Maetzke, Florenz 1994, 381 f. Nr. 8; Cerchiai, 1987, 42f.; ders. 1995, 139.

${ }_{76}$ Zum Apollokult und seiner politischen Bedeutung in Caere vor allem D. Briquel, $1984,219 \mathrm{f}$.

77 Ael. VH 1,20 bezeugt die Verehrung des Apollo in Pyrgi. Weihungen an den chthonischen Gott Suri, der mit Apollo gleichgesetzt wurde, fanden sich südöstlich des Heiligtums der Leukothea-Ilizia; M. Cristofani, s. v. Pirgi, BTCG XIII, Pisa-Rom 1994, 582; B. d'Agostino, Aspetti della funzione politica di Apollo in area tirrenica, in: Culti della Campani antica, 119.

78 L. Cerchiai, Le officine etrusco-corinzie di Pontecagnano, Neapel 1990 (AION Arch. St. Ant. Quad. 6) 133 ff., identifizierte zwei derartige Werkstätten, die im Stil der Meister aus Caere und Vulci arbeiteten. Zu den daraus erschließbaren wirtschaftlichen wie politischen Kontakten der Oberschicht von Pontecagnano mit den Zentren Südetruriens, namentlich Vulci, Veji und Caere, Cerchiai, 1987, 45. 
Westen und Süden vor ${ }^{79}$. Im Westen reichte in antiker Zeit ein Meeresarm nahe an diese Erhebung heran, im Osten bildete der Sarno eine natürliche Begrenzung, während es im Norden keinen deutlichen topographischen Abschluß gab. Erschwert wurde eine Besiedlung durch den porösen Untergrund, der keine Gewinnung von Grundwasser aus Brunnen erlaubte und es daher notwendig machte, Niederschlagswasser zu sammeln oder mühsam Flußwasser herbei zu schaffen ${ }^{80}$.

Ein entscheidender Vorteil dieses Siedlungsplatzes gegenüber dem Sarnotal war allerdings sein natürlicher Schutz. Diesen suchten wohl jene Leute, die bereits im 7. Jh. einfache Hütten auf dem südlichen Sporn des Höhenrükkens, wenig westlich bzw. nördlich des späteren Forum Triangulare, errichteten $^{81}$. Sehr gut war auch die verkehrstechnische Lage des Siedlungshügels: das Mündungsgebiet des Sarno und ein flacher Strand unmittelbar unter dem westlichen Steilabfall schufen zwei natürlich geschützte Schiffslandeplätze ${ }^{82}$; darüber hinaus kreuzten sich im Bereich des späteren Stadtgebietes von Pompeji zwei der wichtigsten Landverbindungen des antiken Kampanien, die Küstenstraße von Cumae zur Sorrentinischen Halbinsel und der Verkehrsweg von Nola bzw. aus dem Sarnotal (mit seiner Hauptsiedlung Nuceria) zur Küste. Die Kombination dieser günstigen topographischen Gegebenheiten führte wohl zur Entstehung einer größeren, auch den zentralen und westlichen Bereich der Hügelkuppe einschließenden Siedlung.

Nahe dem westlichen Steilabfall des Siedlungshügels von Pompeji wurden im späten 7. und frühen 6. Jh. hergestellte Gefäße gefunden. Bei den ältesten Funden handelt es sich um Buccherokeramik capuaner Machart, zu Beginn des 6. Jh.s entstanden die ältesten im griechischen Mutterland hergestellten Gefäße aus Korinth und in geringerer Zahl auch von der Peloponnes ${ }^{83}$. Da diese in einem Fall in einem wohl im 4. Jh. angelegten Votivdepot gefunden wurden und darüber hinaus die selben Formen wie die später mit Sicherheit als Weihegaben im hier gelegenen Apollonheiligtum dargebrachte Keramik aufweisen, ist es wahrscheinlich, daß hier bereits seit dem frühen 6 . Jh. ein

79 Eschebach, Pompeji, 94 Abb. 37.

${ }^{80}$ Eschebach, Pompeji, 16.

81 Jedenfalls vor der zweiten Hälfte des 7. Jh. wurde unmittelbar westlich des späteren Forum Triangulare, an der Stelle des Hauses Regio VIII, 2, 38-39 („Haus Joseph II"), eine einfache Hütte aus Holz errichtet; P Carafa, What was Pompeii before 200 B.C.? Excavations in the House of Joseph II, in the Triangular Forum and in the House of the Wedding of Hercules, in: Sequence and Space in Pompeii, Hgg. S. E. Bon/R. Jones, Oxford 1997 (Oxbow Monograph 77) 15-20, zum datierenden Material 16f. mit Anm. 2 u. 3. Spuren dieser ältesten Wohnbebauung Pompejis wurden auch wenig nördlich des Forum Triangulare, im Bereich des späteren Isistempels gefunden und von O. Elia, Osservazioni sull'urbanistica di Pompei, il nucleo originario dell'impianto, in: Studi sulla città antica, Bologna 1970, 183-190, angezeigt. Aufgrund der nur vorläufigen Publikation des Befundes können diese allerdings bisher nicht eindeutig datiert werden, wie zuletzt H. W. Horsnaes, From the Iron Age to Pompei. Urbanization in southern Campania - a case study, in: Urbanization in the Mediterranean in the 9th to 6th centuries B. C., Hgg. H. Damgaard Andersen/H. W. Horsnaes/S. Houby-Nielsen/A. Rathje, Kopenhagen 1997 (Acta Hyperborea 7) 198f., betonte.

82 Strab. 5,4,8; zum Küstenverlauf in antiker Zeit und zum Hafen von Pompeji L. Eschebach, Hafenstadt Pompeji, AW 20,1 (1989) 40-54; Eschebach, Pompeji, 1 Anm. 5 u. $94 \mathrm{ff}$.

83 St. de Caro, Saggi nell'area del tempio di Apollo a Pompei, Neapel 1986, $59 \mathrm{ff}$., nennt die Fragmente von 20 Buccherogefäßen, einen italo-geometrischer Stamnos pithekussanisch-cumanischer Tradition, 21 Fragmente korinthischer Keramik, sowie eine Kylix und eine Schale lakonischer Machart. 
Kultort des Apollo bzw. eines diesem entsprechenden italischen Gottes unterhalten wurde ${ }^{84}$.

Im zweiten Viertel des 6 . Jh. wurde in diesem Heiligtum ein erster monumentaler Kultbau errichtet. Es handelte sich dabei um ein hölzernes Gebäude dessen Dach mit Terrakotten verziert war, die wahrscheinlich in einer cumanischen Werkstatt hergestellt wurden ${ }^{85}$. Die Errichtung dieses Baus bezeugt den öffentlichen Charakter des Heiligtums und ist Ausdruck der politischen Organisation der Siedlungsgemeinschaft.

Das Heiligtum lag auf jener langgestreckten, ebenen Zunge, die vom westliche Ausläufer des von Norden nach Süden allmählich absinkenden Lavastromes gebildet wurde und im Westen und Süden steil zum Meer hin abfiel $^{86}$. Im Osten wurde diese Fläche durch eine deutliche Bodenwelle begrenzt und vom Bereich der ältesten Hüttenbauten am bzw. nahe dem späteren Forum Triangulare abgesetzt ${ }^{87}$. Gleichzeitig war sie von dem unterhalb des westlichen Steilabfalls gelegenen Seehafen aus leicht zugänglich ${ }^{88}$. Dieses unbebaute Areal eignete sich daher gut für eine Nutzung als öffentlicher Marktplatz, eine Funktion, die später in samnitischer und schließlich römischer Zeit durch das hier gelegene Forum Civile übernommen und erweitert wurde ${ }^{89}$.

Der erste profane Monumentalbau Pompejis, ein großer Mauerring, entstand ebenfalls bereits in der ersten Hälfte des 6 . Jh.s und umschloß das gesamte Areal zwischen dem Steilabfall im Westen und der späteren Porta Nuceriana ${ }^{90}$, also die Hüttensiedlung, das Areal um das Apollonheiligtum, sowie die verkehrstechnisch überaus bedeutende Kreuzung der Küstenstraße mit den Verkehrsverbindungen aus dem Hinterland zur Küste.

\section{Die Italiker im Sarnotal und auf der Sorrentinischen Halbinsel und die Etrusker in Pompeji}

Ab der Wende des 7. zum 6. Jh.s lassen sich im weiteren Umkreis von Pompeji, an der Nordküste der Sorrentinischen Halbinsel und im Sarnotal, an mehreren Stellen neue Siedlungen nachweisen (Karte 1) ${ }^{91}$. Ob allerdings die

${ }^{84} \mathrm{Zu}$ diesem Votivdepot, das östlich des späteren Podiumstempels gefunden wurde, St. de Caro, 1986, s. o. Anm. 83, 9; auch er spricht sich S. 20, aufgrund des ältesten Keramikmaterials für eine durchgehende Nutzung des Areals als Apolloheiligtum seit dem frühen 6. Jh. aus.

${ }^{85}$ Erhalten hat sich von diesen Verzierungen allerdings nur ein Blattrandantefix; St. de Caro, 1986, s. o. Anm. 83, 21, 37 Kat.-Nr. 14.

${ }^{86}$ Eschebach, Pompeji, 16 ff. mit Abb. 7.

87 Diese Geländeschwelle verläuft an der Ostseite der späteren Via di Eumachia, die sich in ihrer südlichen Verlängerung in der Ostseite des Vico dei Dodici Dei fortsetzt. H. Eschebach, RM 89 (1982) 298 Anm. 107; Taf. 104,1; 105,1; 279 Abb. 25; Eschebach, Pompeji, 18.

88 Eschebach, Pompeji, 94f. Abb. 37 mit Darstellung des antiken Küstenverlaufs und Beschreibung der Straßenverbindungen des Seehafens mit dem Stadtgebiet.

${ }^{89}$ Eschebach, Pompeji, $65 \mathrm{f}$.

90 St. de Caro, Nuove indagini sulle fortificazioni di Pompei, AION 7 (1985) $86 \mathrm{~m}$. Anm. 43., zusammenfassend zu Technik und Datierung S. 104 f., fig. 27; vgl. auch St. de Caro, Lo sviluppo urbanistico di Pompei, in: Atti Magna Grecia, 1992, 69. Zur Datierung zuletzt H. W. Horsnaes, 1997, s. o. Anm. 81, 199 f., die sich kritisch zum Zeitansatz in der ersten Hälfte des 6. Jh. äußert, sowie F. Zevi, I Greci, gli Etruschi, il Sele, in: Culti della Campania, 3, der die Errichtung der Mauer aufgrund des stratigraphischen Befundes (mittelkorinthische Keramik) im erste Viertel des 6. Jh. ansetzt.

91 H. W. Horsnaes, 1997, s. o. Anm. 81, 205-209. 
Bezeichnung der wichtigsten Siedlung des Sarnotals als Nuceria, „Neustadt“, mit diesem Prozeß der Ausbildung größerer geschlossener Siedlungsgemeinschaften in archaischer Zeit zu verbinden ist, oder aber dieser Ortsname erst im Zuge des Urbanisierungsprozesses unter den Samniten gebildet wurde, ist nicht eindeutig zu entscheiden ${ }^{92}$. Monumentale Bauten lassen sich jedenfalls in der ersten Hälfte des 6. Jh.s außerhalb von Pompeji nicht nachweisen.

Den vermögenden Bewohnern dieser italischen Siedlungsgemeinschaften wurden nach ihrem Tod neben Erzeugnissen lokaler Produktion auch Luxus- und Gebrauchsgegenstände etruskischer (vor allem capuanischer) sowie griechischer Machart mit ins Grab gegeben ${ }^{93}$. Gleichzeitig bedienten sie sich der oskischen Schriftsprache, wobei sie ein eigenes Alphabet (und nicht etwa etruskische oder griechische Buchstaben) verwendeten ${ }^{94}$.

Ein gutes Beispiel eines solchen Angehörigen der italischen Oberschicht des Sarnotals liefert ein um die Mitte des 6. Jh.s in Nuceria bestatteter Mann. Diesem wurden die zum gepflegten Weinkonsum notwendigen Gefäße mit ins Grab gegeben ${ }^{95}$. Während auf einem Weinkrug die oskische Besitzerinschrift // bruties // esum, ,ich gehöre Brutius“, angebracht ist ${ }^{96}$, wurde auf den Gefäßkörper einer im selben Grab gefundenen Trinkschale in großen Lettern des euböischen, in Cumae verwendeten Alphabets die Aufschrift ảoíotov geritzt $^{97}$. Männer wie der hier Bestattete kannten und schätzten also die über

92 Zur sprachlichen Form des Namens Nuceria vor allem G. Calzecchi Onesti, Ocr e Acr nella toponomastica dell'Italia antica, StEtr 49 (1981) 165ff., sowie Briquel, 1984, 575f. Auch der Name der nördlich des Vesuv gelegenen Siedlung Nola ist wohl von diesem Stamm abzuleiten und daher mit einem Prozeß des Zusammensiedelns zu verbinden. Zum archäologischen Befund in Nuceria W. Johannowsky, Nuovi rinvenimenti a Nuceria Alfaterna, in: La regione sotterata dal Vesuvio. Studi e prospettive, Atti del conv. int. 1979, Neapel 1982, 837 f.; E. Esposito, L'Ager Nucerinus. Note storiche e topografiche, RendAccNap 59 (1984) 221 ff., sowie A. Pontrandolfo, s. v. Nocera Superiore, in: BTCG XII, Rom-Pisa 1993, 351 ff.; zu den Funden in Nola Cerchiai, 1995, 128. $135 \mathrm{ff}$. Den Zeitpunkt der Gründung dieser „Neustadt“ setzen B. d'Agostino, 1988, s. o. Anm. 1, 566 f. und Cerchiai, 1995, 128, im späten 7. bzw. frühen 6. Jh. an. Für eine Bezeichnung als „Neustadt“ im Zuge der samnitischen Bauaktivitäten St. de Caro, Lo sviluppo urbanistico di Pompei, in: Atti Magna Grecia, 1992, 75.

93 Die in Capua überaus beliebten fibule a ghiande wurden sowohl in Nuceria, Stabiae und Sorrent, als auch an der Südküste der Sorrentinischen Halbinsel in Vietri sul mare gefunden; C. A. Livadie, La situazione in Campania, in: Il commercio etrusco arcaico, Quaderni del centro di studio per l'archeologia etrusco-italica 1985, 130 mit Anm. 28. Etruskische Amphoren: C. A. Livadie, 1985, 143 Nr. C 5 (Vico Equense, erste Hälfte 6. Jh.), 142 Nr. B 33 (Nocera, erstes Viertel 6. Jh.), 143 Nr. D 2 (Stabiae, Mitte 6. Jh.). Die ältesten, bisher nur in Vorberichten angezeigten griechischen Gefäße des 7. und 6. Jh.s wurden in St. Marco nahe Stabiae gefunden. Dazu Cerchiai, 1987, 46 f., mit Nennung des analogen Befundes in Nola, sowie jüngst H. W. Horsnaes, 1997, s. o. Anm. 81, 207. Vgl. auch die schon oben Anm. 83 genannte, in Pithekussai bzw. Cumae hergestellte italogeometrische Keramik, die sowohl in Pompeji wie auch in Gräbern des Sarnotals gefunden wurde.

${ }_{94} \mathrm{Zu}$ diesem schon oben bes. Anm. 75 .

95 Grab Nr. 32 einer im Jahr 1964 von V. Panebianco in der loc. Pareti in Nocera Superiore ergrabenen Nekropole. Die Beigaben wurden von G. Colonna, Nuceria Alfaterna, StEtr 42 (1974) 379-386, publiziert.

${ }_{96}$ Die Lesung bruties bei R. Wallace, 1990, s. o. Anm. 75, sowie M. Cristofani, 1994, s. o. Anm. 75, 381 Nr. 6. Anders G. Colonna, 1974, s. o. Anm. 95, 386, der den Besitzernamen fraties liest.

97 G. Colonna, 1974, s. o. Anm. 95, 379 f.; M. Cristofani, Presenze etrusche tra Stabia e Pontecagnano, Atti Magna Grecia, 1992, 65 Anm. 23. Die Aufschrift wird allgemein als Personenname gedeutet (vgl. etwa den bei Strab. 6,1,9 [C 260] genannten Kithairo-

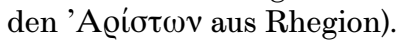


Capua und Cumae vermittelten etruskisch-griechischen Trinksitten, blieben aber gleichzeitig ihrer italischen Lebensart treu und brachten dies durch die Verwendung der oskischen Schriftsprache zum Ausdruck ${ }^{98}$. Seit dem Beginn des 6. Jh.s gab es somit an der Küste der Sorrentinischen Halbinsel und im Sarnotal eine Klientel für etruskische sowie griechische Luxusgüter und Gebrauchsgegenstände.

Die bauliche Erweiterung der Siedlung auf dem verkehrstechnisch günstig gelegenen Hügel von Pompeji ist wohl als Reaktion auf diesen neuen Absatzmarkt zu erklären, wobei sich die Frage stellt, wer für diesen strukturellen Wandel verantwortlich war. Der große Mauerring, für den es in Kampanien keine älteren oder zeitgleichen Vergleichsbeispiele gibt ${ }^{99}$, entspricht formal am ehesten jenen künstlichen Befestigungsanlagen, mit denen die Griechen ihre Handelsstützpunkte sicherten ${ }^{100}$, während mit Terrakottaplatten verzierte Kultbauten wie jener in Pompeji sowohl im etruskischen Capua wie auch im griechischen Cumae beliebt waren.

Neben diesem archäologischen Befund bietet die literarische Überlieferung in Verbindung mit epigraphischen Zeugnissen eine Quellenbasis, die weitere Überlegungen zur politischen Situation Pompejis im 6. Jh. und damit zur Herkunft der für den strukturellen Wandel der Siedlung verantwortlichen Personen ermöglicht. Strabon 5,4,8 (C 247) unterscheidet in Pompeji (und übereinstimmend im nahegelegenen Herkulaneum) drei unterschiedliche Phasen politischer Herrschaft, nämlich eine italische, dann eine etruskischpelasgische und schließlich eine samnitische. Mit D. Briquel ist diese Tradition am ehesten als eine lokale Überlieferung der pompejianischen Frühgeschichte zu sehen, die eine tatsächliche Phase etruskischer Herrschaft in Pompeji reflektiert ${ }^{101}$. Eine Stütze findet diese Hypothese in etruskischen Gefäßaufschriften des späteren 6. und frühen 5. Jh.s aus dem Apollonheiligtum von Pompeji, die im Dialekt von Caere bzw. Veji abgefaßt sind ${ }^{102}$. Jüngst wurden darüber hinaus derart beschriftete Gefäße auch im Siedlungsbereich

98 Cerchiai, 1987, 47, schloß daraus auf eine Freundschaftsbeziehung in der Art der griechischen xenia dieses italischen Aristokraten mit seinen Standesgenossen in Cumae; als Analogie führt er die Situation in Pontecagnano an, wo Kontakte der ansässigen Etrusker mit dem achäischen Poseidonia erschließbar sind.

99 Die jüngsten Grabungen im Bereich der nördlichen Ummauerung der Unterstadt von Cumae haben eine Datierung in das späte 6. Jh., also in die Herrschaftszeit des Aristodemos, bestätigt; siehe B. d'Agostino/F. Fratta, Gli scavi dell'I.U.O. a Cuma negli anni 1994-95, AION N.S. 2 (1995) 201-209. Diese Phase der Ummauerung der Unterstadt scheint auch schon von W. Johannovsky, Problemi relativi a Cuma arcaica, in: Contribution à l'etude de la Societé et de la Colonisation Ėubéens, Neapel 1975 (Cah. du Centre J. Berard) 98, im östlichen Bereich des Stadtgebietes festgestellt worden zu sein.

100 Die Errichtung eines derartigen $\tau \varepsilon \hat{\chi} \chi 0 \varsigma$ bezeugt Strab. 6,1,1 (C 252) etwa für die selbe Zeit wie die Errichtung des Mauerrings von Pompeji als Maßnahme der sybaritischen Siedler an der lukanischen Küste. Zur Art dieser Befestigung und ihrer Lokalisierung schon ausführlich oben bezüglich der Gründung von Poseidonia (Literaturverweise in Anm. 8).

101 Briquel, 1984, $567 \mathrm{f}$., erkennt als wahrscheinliche Quelle dieser Tradition den ephesischen Geographen Artemidor, der diese vielleicht sogar am Ort selbst gehört und aufgezeichnet habe.

102 G. Colonna, Nuovi dati epigrafici sulla protostoria della Campania, in: Atti della XVII. riunione scientifica dell'instituto italiano di preistoria e protostoria, Florenz 1975, 159; ders., L'etruscizità della Campania meridionale, in: La presenza etrusca nella Campania meridionale, Atti delle giornate di studio a Salerno e Pontecagnano 1990, Hgg. P. Gastaldi/G. Maetzke, Florenz 1994, 360 f. 
Pompejis gefunden ${ }^{103}$. Diese Ritzinschriften bezeugen eine dauerhafte Anwesenheit von Etruskern des südlichen Mutterlandes in Pompeji, können allerdings den Grad der politischen Einflußnahme derselben nicht erweisen. Einen solchen Hinweis auf eine politische Einflußnahme von Caeretanern in Pompeji gibt dafür die Art des hier vollzogenen Apollonkults. L. Cerchiai hat diesen jüngst mit der stark vom griechischen Delphi beeinflußten Verehrung des Gottes in Caere in Verbindung gebracht ${ }^{104}$. Entsprechend der politischen Bedeutung des Apollonkults in Caere wäre die Einführung seiner Verehrung in Pompeji als Zeichen einer etruskischen Machtübernahme zu interpretie$\mathrm{ren}^{105}$. Von diesen Etruskern wäre auch der in der literarischen Überlieferung erhaltene Name der Bewohner des Sarnotals Sarrastrae gebildet worden, der als etruskisierte Form des italischen Sarasnes gelten kann ${ }^{106}$.

Aufgrund dieser literarischen Zeugnisse und auf Basis der monumentalen Bauaktivitäten in der ersten Hälfte des 6. Jh. ist es plausibel, daß Pompeji auf Initiative von Etruskern aus dem südlichen Mutterland, vor allem aus Caere, von einer einfachen Hüttensiedlung zu einem ummauerten Handelsplatz mit einem Apollonheiligtum gemacht wurde. An dem nun verstärkt stattfindenden Warenaustausch waren wohl zur See anreisende etruskische und griechische Händler ebenso beteiligt wie Kaufleute aus dem Hinterland (aus dem Sarnotal, aus Nola und vielleicht auch aus dem etruskischen Capua) und die ansässige italische Bevölkerung. Geeigneter Ort dieser Handelsaktivitäten war der an der Wende vom 7 . zum 6 . Jh. wohl noch weitgehend unbebaute westliche Bereich des Siedlungshügels. Die Einrichtung eines Heiligtums an dieser Stelle und insbesondere seine Ausstattung mit einem monumentalen Kultgebäude war eine öffentliche Baumaßnahme und damit ein politischer Akt, mit dem wohl auf den Bedeutungswandel Pompejis von einer einfachen Wohnsiedlung zu einem wichtigen Handelsplatz reagiert wurde.

\section{Zwischenergebnis}

Handelsinteressen waren ein entscheidender Faktor der griechischen Niederlassungen an der Nord- und Südgrenze des poseidoniatischen Territoriums. Die Siedlung am Kap Agropoli dürfte dabei primär als Station für Händler zur See gegründet worden sein, sollte jedoch wohl kaum dem Warenaustausch mit dem italischen Hinterland dienen. Dem entsprechen die Weihegaben im hier eingerichteten Heiligtum, die am ehesten von den ansässigen Griechen sowie von griechischen und vielleicht etruskischen Händlern gestiftet wurden. Diese Niederlassung und wohl auch jene an der Selemündung sind mit den handelspolitischen Interessen von Sybaris im späteren 7. und in der ersten Hälfte des 6. Jh.s v. Chr. zu verbinden, das sich hier Stützpunkte auf der Seehandelsroute entlang der italischen Westküste sichern wollte ${ }^{107}$.

Ein Warenaustausch an dem neben Etruskern und Griechen auch die Italiker beteiligt waren, ist in Pompeji, Pontecagnano sowie an der Mündung des Sele wahrscheinlich. Diese Handelsaktivitäten wurden wohl seit dem späten

103 H. Blank, Rivista di epigrafia etrusca. Pompeii, StEtr 61 (1996) 321-323; vgl. auch F. Zevi, 1998, s. o. Anm. 90, 8.

${ }^{104}$ L. Cerchiai, Aspetti della funzione politica di Apollo in area tirrenica, in: Culti della Campania antica, 123-128.

105 B. d'Agostino, 1998, s. o. Anm. 77, 122 f.

106 G. Radke, s. v. Sarnus, Der Kleine Pauly IV, München 1972, 1559.

107 Dazu schon oben bes. Anm. 53 und 56-58. 
7. bzw. seit dem frühen 6. Jh. von öffentlicher Seite durch das Bereitstellen von Lande- Stapel- und Marktplätzen gefördert, und zum Teil auch durch die Errichtung entsprechender Bauten (Säulenhallen in Pontecagnano und an der Selemündung, schützender Mauerring in Pompeji) unterstützt. $\mathrm{Zu}$ diesen öffentlichen Einrichtungen gehörten auch Kultstätten am jeweiligen Ort des Warenaustauschs, wobei in Pontecagnano eine Stelle am Rand des eigentlichen Siedlungsgebietes, in Pompeji wahrscheinlich eine Freifläche abseits der Hüttensiedlung, gewählt wurde. Das Heraion an der Selemündung lag ebenfalls außerhalb geschlossener Siedlungsräume ${ }^{108}$ und gleichzeitig an der Grenze des von Griechen beanspruchten Territoriums. Es wurden also Plätze bevorzugt, die gewissermaßen auf „neutralem Boden“ zwischen der Wohnsiedlung und der Landestelle zugereister Händler lagen ${ }^{109}$. Damit entsprechen diese Handelsplätze prinzipiell den im griechischen Mutterland litera-

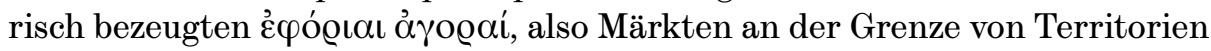
unterschiedlicher politischer Gemeinschaften ${ }^{110}$.

Einer rechtlichen Gleichstellung der Handelspartner an diesen Orten des Warenaustauschs entspricht auf sakraler Ebene die Verehrung von Gottheiten, die allen Besuchern vertraut waren; in den hier untersuchten Fällen sind dies Hera (an der Mündung des Sele) und Apollo (Pontecagnano, wahrscheinlich auch in Pompeji). Diese Gottheiten konnten als Garanten der Rechtmäßigkeit der abgeschlossenen Geschäfte dienen und somit das für Handelskontakte unablässige Vertrauensverhältnis zwischen Käufer und Verkäufer herstellen. Diese Vorstellung ist von griechischer Seite für den Kult des Zeus Xenios, der seit dem späten 6. Jh. auch in Poseidonia verehrt wurde ${ }^{111}$, gut bezeugt. Dieser Kult war Ausdruck einer staatlich garantierten Rechtssicherheit der $\xi \dot{\varepsilon} v o \iota$ und wurde in diesem Sinn auch in den Gesetzen des Charondas von Katane definiert ${ }^{112}$. Dabei schloß der umfassende Charakter des Zeus

108 Eine intensive Nutzung dieses Areals nördlich bzw. östlich des Heraheiligtums in antiker Zeit ist durch reiche Oberflächenfunde von Gebrauchskeramik und Ziegeln bezeugt, die bisher allerdings nur vorläufig angezeigt wurden; Greco, 1992, 250. Die aus diesen Funden erschließbaren baulichen Einrichtungen sind mit einer Nutzung der lagunenartigen Verzweigungen des Sele als natürliche Schiffslandeplätze zu verbinden, von denen oben (vgl. bes. Anm. 68) bereits die Rede war.

109 Eine solche Lage ist auch für mehrere außerstädtische Heiligtümern der Etrusker und Griechen außerhalb Kampaniens nachgewiesen oder doch mit gewisser Wahrscheinlichkeit erschließbar. Beispiele wurden von G. Colonna, 1985, s. o. Anm. 42, 127, zusammengestellt. Vgl. auch F. de Polignac, Mediation, competition and sovereignty: the evolution of rural sanctuaries in geometic Greece, in: Placing the gods. Sanctuaries and sacred space in ancient Greece, Hgg. S. Alcock/R. Osborne, Oxford 1994, 6f., mit dem Hinweis auf die an bedeutenden Handelsrouten angelegten griechischen Heiligtümer.

$110 \mathrm{Zu}$ diesen vor allem von Dem. 23,37-39 und Poll. 9,8-9 angesprochenen,Grenzmärkten' G. Daverio Rocchi, Frontiera e confini nella Grecia antica, Rom 1988, 30 (zu Demostehenes), 208 (zu Pollux) und 225.

$111 \mathrm{Zu}$ diesem Zeitpunkt wurde ein Silberbarren als $\tau \tilde{0} \Delta$ ı̀̀ $\xi \varepsilon i ́ v o$, als „(Besitz) des Zeus Xenios“, gekennzeichnet. A. M. Ardovino, Nuovi oggetti sacri con iscrizioni in alfabeto acheo, Arch. Class. 23 (1980) 65f.; R. Arena, 1996, s. o. Anm. 23, 48 Nr. 23.

112 Charondas ap. Stob. 4,40. Zusammenstellung und Diskussion aller literarischen Belege des Zeus-Xenios-Kults schon bei L. R. Farnell, The Cults of the Greek States, Vol.

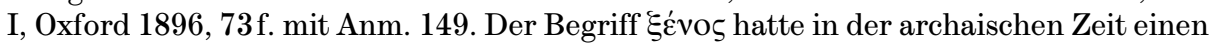
sehr allgemeinen Charakter und meinte all jene Personen, die nicht dauerhaft im Stadt-

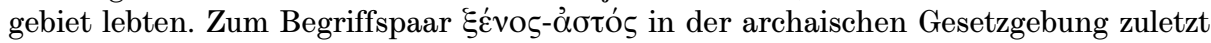
Nomima, Recueil d'inscriptions politiques et juridiques de l'archaisme grec, Bd. I, Hgg. H. v. Effenterre/F. Ruzé, Rom 1994, 29. 
Xenios Griechen wie Nichtgriechen gleichermaßen ein ${ }^{113}$. Entscheidend für die Wahl der Gottheit war also deren Akzeptanz durch Angehörige der unterschiedlichen Volksgruppen. Dieser Anforderung entsprachen vor allem Götter, die eine Fülle von Aspekten in sich vereinten, wie Hera, Apollo oder Zeus. Dabei entschied sich die ansässige Bevölkerung wohl letztlich für eine Gottheit, die bereits fest in ihrem religiösen Leben verwurzelt war. So wurde etwa Hera auch an der Mündung des Sele als Stadtgöttin verehrt, also in der selben Weise wie in Poseidonia selbst ${ }^{114}$. Der caeretaner König Thefarie Velianas wiederum, der in Pyrgi, dem Hafenplatz von Caere, ein Heiligtum für UniHera-Astarte stiftete, tat dies aufgrund seiner persönlichen Beziehung zu dieser Göttin, wie es in der Stiftungsurkunde vermerkt ist ${ }^{115}$. Analog dazu ist es anzunehmen, daß auch die politisch Verantwortlichen in Pontecagnano und Pompeji eine klare Vorstellung davon hatten, was sie mit der Einführung eines Kultes Apollos bezweckten.

\section{Die ErRichtung öfFentlicher Grossbauten und der Austausch von ARChITEKTUR- und DeKoRATIONSFormen}

\section{Pompeji}

Im letzten Drittel des 6. Jh.s erfuhr das Heiligtum des Apollo in Pompeji eine umfassende Neugestaltung. Nun wurden ein Altar sowie ein Tempel im etruskisch-italischen Typ errichtet, ein Holzbau auf einem Steinpodium, dessen Dachverzierung stilistisch in der Tradition des Kunstschaffens in Cumae steht ${ }^{116}$.

Im Bereich der alten Hüttensiedlung, auf dem späteren Forum Triangulare, wurde schon um die Mitte des 6. Jh.s ein Steintempel errichtet, den seine steinernen kannelierten Säulen und Kapitelle, der Stufenbau sowie die Grundform des Peripteros, als griechischen Kultbau definieren ${ }^{117}$. Die Maße dieses Tempels finden ihre eheste Entsprechung in gleichartigen Kultbauten Altitaliens ${ }^{118}$. Die Form der dorischen Kapitelle dieses griechischen Tempels

113 In jener Geschichte, die Odysseus im 14. Gesang der Odyssee dem Schweinehirten Eumaios erzählt, berichtet er auch von seinen angeblichen Abenteuern in Ägypten, wobei er vom dortigen König vor dem Tod gerettet worden sein, da dieser das von Zeus

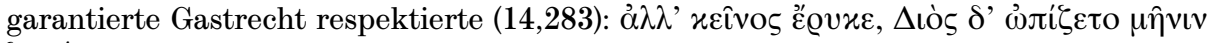
छcıvíov.

114 Siehe oben Anm. 41.

115 Zum Heiligtum von Pyrgi schon oben Anm. 42 u. 77; zur Stiftungsurkunde des Thefarie weiters G. Garbini, L'iscrizione di Pyrgi, RSF XVII (1989) 179-187; G. Colonna, Rivista di epigrafia etrusca. Pyrgi, StEtr 61 (1989/90) 313-324.

116 St. de Caro, 1986, s. o. Anm. 83, 21 und bes. 37 ff. (Katalogteil); Cerchiai, 1995, $131 \mathrm{f}$.

117 Nachgrabungen unter Leitung von J. de Waele sollen neue Erkenntnisse über die Zeitstellung erbringen und eine architektonische Rekonstruktion dieses Kultbaus ermöglichen. Erste Ergebnisse dieser Untersuchungen wurden als The Doric Temple on the Forum Triangulare at Pompei, OpPomp III (1993) 105-118 [engl. Üs. eines Beitrags in Hermeneus 54 (1982) 27-35] publiziert (vgl. auch StPomp 1995); derzeit bleiben jedoch die Arbeiten von F. v. Duhn/L. Jacobi, Der griechische Tempel in Pompeji, Heidelberg 1890; J. Richardson jun., The archaic doric temple of Pompeii, PP 29 (1974) 281-290; V. Kockel, AA 1986, 462 ff.; Mertens, 1993, 173 und zuletzt Cerchiai, 1995, 134f., grundlegend.

118 In den allgemeinen Proportionen und der Säulenzahl sind am ehesten die Tavole Palatine sowie der Kardaki-Tempel auf Korfu vergleichbar, wie Mertens, 1993, 173, zeigte. J. A. de Waele, The ,doric' temple on the Forum Triangulare in Pompeii, OpPomp 
in Pompeji folgt allerdings Vorbildern aus Poseidonia ${ }^{119}$. Im südlichen Stadtheiligtum von Poseidonia wurden darüber hinaus im späten 6. Jh. auch mehrere Gebäude errichtet, deren Dächer und Gebälk in der selben Art verziert sind wie jenes des griechischen Tempels in Pompeji ${ }^{120}$. Diese Dekoration steht ganz allgemein in einer von cumanischen Meistern entwickelten kampanischen Tradition ${ }^{121}$, zeigt aber auch Elemente, die außerhalb von Pompeji und Poseidonia bisher nicht bezeugt sind ${ }^{122}$. Es ist daher wahrscheinlich, daß dieselbe kampanische Werkstatt für die Herstellung dieser Dachverzierungen in beiden Siedlungen verantwortlich war ${ }^{123}$.

In Pompeji bedeutete die Übernahme poseidoniatischer Steinarchitektur ein neues Element in einem Formenkanon öffentlicher Großbauten, der sich bis dahin ausschließlich an den Vorbildern Capua und Cumae orientiert hatte. Eine noch radikalere Neuerung war die Verwendung etruskisch-kampanischer Dachverzierungen in Poseidonia, da damit erstmals ein Kunststil gewählt wurde, der nicht in der eigenen achäisch-großgriechischen Tradition stand.

\section{Die Dekoration öffentlicher Großbauten in Fratte bei Salerno}

In Fratte, einem Vorort des heutigen Salerno (Karte 1), weisen Bestattungen eine dauerhafte Ansiedlung von Italikern seit dem ersten Viertel des 6. Jh.s nach $^{124}$. Ab dem dritten Viertel des 6 . Jh.s v. Chr. befand sich hier eine Siedlung deren monumentale bauliche Gestaltung städtischen Charakter zeigt. Sie lag etwa drei Kilometer von der heutigen Küste entfernt auf einem Hügel über dem Fluß Irno und damit an jener Landverbindung, die das Sarnotal mit dem Golf von Salerno verband und weiter nach Süden in den ager Picentinus und nach Poseidonia führte ${ }^{125}$. Der aus Ton gefertigte Architekturdekor der bedeutenderen Gebäude wurde hier zunächst, im dritten Viertel des 6. Jh.s, aus Capua importiert und bald darauf in dieser Tradition auch von ansässigen Meistern hergestellt ${ }^{126}$. Im letzten Viertel des 6. Jh.s kamen dazu cumanische Erzeugnisse ${ }^{127}$.

III (1993) 111, vergleicht den Bau mit Tempel B von Pyrgi und Tempel II von Satricum und stellt das Kultgebäude in Pompeji in die etruskisch-italische Architekturtradition.

119 D. Mertens, 1993, 173, der auch auf formal entsprechende Beispiele aus Vico Equense bei Sorrent hinweist (Taf. 92, 3-6).

120 D. Gasparri, BdA, N.S. 74 (1992) 65ff.; Mertens, 1993, 172; ders., 1994, s. o. Anm. 6, $209 \mathrm{ff}$.

121 D. Mertens, 1994, s. o. Anm. 6, 195-219, 210 f., Taf. VI,2-3 u. VIII, 1-2; D. Gasparri, 1992, s. o. Anm. 120, $68 \mathrm{ff}$.

122 D. Mertens, 1994, s. o. Anm. 6, 209 f., Taf. V,1.

123 Dies findet eine Stütze darin, daß der Ton eines der poseidoniatischen Dächer stark mit vulkanischem Sand vermischt ist, also mit hoher Wahrscheinlichkeit in Kampanien hergestellt wurde. D. Mertens, 1994, s. o. Anm. 6, 211, Taf. VII; D. Gasparri, 1992, s. o. Anm. 120, 72.

124 Die Befunde der frühesten Gräber wurden zuletzt von D. Donnarumma/L. Tomay, I corredi di VI e V sec. a. C., in: Fratte, 1990, 207-211, zusammengestellt und diskutiert.

125 Diese Landverbindung beschreibt Strab. 5,4,13, der die Entfernung von Pompei über Nuceria zum Golf von Salerno mit 120 Stadien angibt; dazu L. Vecchio, 1990, s. o. Anm. 3, 18-21, mit weiterer Literatur. Allgemein zu den topographischen Gegebenheiten auch T. Cinquantaquattro, Dinamiche insediative nell'agro picentino dalla protostoria all'età ellenistica, AION 14 (1992) 245 und Karte auf S. 246.

126 G. Greco, I Materiali dai vecchi scavi dell'abitato. 1. Terrecotte architettoniche, in: Fratte, 1990, 59 ff.; Import aus Capua: S. 77 Nr. 1, Fig. 61; lokales Erzeugnis: Nr. 2, 
Im frühen 5. Jh. wurde schließlich bei der Verzierung eines Daches auch auf den in Poseidonia entwickelten Stil zurückgegriffen ${ }^{128}$. Ebenfalls poseidoniatisch ist die Form zweier etwa zeitgleich entstandener dorischer Steinkapitelle $^{129}$ sowie eine knapp unterlebensgroße Terrakottaskulptur, wahrscheinlich eine Kultstatue ${ }^{130}$. Dieser Befund entspricht der Situation in Pompeji und macht es wahrscheinlich, daß auch Fratte spätestens seit der Wende vom 6. zum 5. Jh. intensive Kontakte zu Poseidonia unterhielt, was sich auch in der Übernahme poseidoniatischer Architektur- und Dekorationsformen zur Gestaltung öffentlicher Großbauten äußerte.

\section{Ein Kultbau in der italischen Siedlung am Monte Pruno im Hinterland von Poseidonia}

In Poseidonia hergestellte Statuetten und Gefäße wurden gemeinsam mit attischer Keramik nach Eboli sowie durch das Flußtal bei Fonte und über den Monte Pruno nach Sala Consilina im Vallo di Diano verhandelt ${ }^{131}$. Innerhalb dieses Handelsraumes im Hinterland von Poseidonia ist eine in der zweiten Hälfte des 6. Jh.s am Monte Pruno gegründete Siedlung (Karte 2) von besonderer Bedeutung, da hier an der Wende vom 6. zum 5. Jh. ein monumentaler Kultbau errichtet wurde, dessen Ziegel und Antefixe formal jenen des großen Tempels im Heraion am Sele entsprechen ${ }^{132}$. Auch hier hatten also die Kontakte zum etwa $30 \mathrm{~km}$ westlich gelegenen griechischen Poseidonia Einfluß auf die Gestaltung öffentlicher Großbauten der italischen Siedlungsgemeinschaft.

\section{Zwischenergebnis}

Die eben genannten italischen Siedlungsgemeinschaften konnten im $6 \mathrm{Jh}$. weder auf eine eigene Tradition monumentaler Steinarchitektur zurückgreifen, noch waren sie mit der Anfertigung baulicher Dekorationselemente aus Ton vertraut. Hatten sie sich also grundsätzlich für die Errichtung eines monumentalen öffentlichen Gebäudes entschieden, so mußten zunächst die funktionalen Anforderungen (Verwendungszweck des Gebäudes) bedacht und die notwendigen wirtschaftlichen (Finanzierung) und technischen Voraussetzungen (Verfügbarkeit von Baumaterial und kundigen Handwerkern) geschaffen werden. Prinzipiell standen etruskische und griechische Vorbilder zur Verfügung, wobei auch ästhetisches Empfinden und die mit der Form des Gebäudes bezweckte Repräsentation eine wichtige Rolle bei der Entscheidung

Fig. 62. Aus der folgenden Generation (etwa 520 bis 480) stammen die importierten Antefixe S. 77 Nr. 3, S. 78 Nrn. 15. 16 und die lokalen Erzeugnisse S. 77 Nrn. 4-7, S. 78 Nrn. 8-14.

127 G. Greco, s. o. Anm. 126, 63f., Kat.-Nr. T IV,3, Fig. 67, S. 78 Nr. 15.

128 G. Greco, Frammenti architettonici in pietra, in: Fratte, 1990, 87, Fr. L 1-4, Figg. 128. $129 \mathrm{a}$, b.

129 G. Greco, s. o. Anm .128, 87 f., Fr. L 5.6, Figg. 130. 131.

130 G. Greco, Coroplastica, in: Fratte, 1990, 105 mit Fig. 161, 106 Nr. 1. Zu allen genannten Belegen Cerchiai, 1995, 120.

${ }^{131}$ Ein in Eboli gefundenes Grab enthielt eine im dritten Viertel des 6. Jh.s in Poseidonia hergestellte weibliche Statuette; einem anderen Verstorbenen wurde hier im

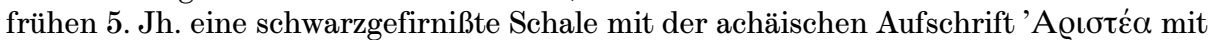
ins Grab gegeben; M. Cipriani, 1990, s. o. Anm. 34, 130 f. Zum Handelsweg von Poseidonia ins Vallo di Diano schon oben Anm. 18.

132 J. de La Genière, Ricerca di abitati antichi in Lucania, Atti e Mem. della Soc. Magna Grecia N.S. VIII (1964) 129-138. 
für einen bestimmten Stil gespielt haben dürften ${ }^{133}$. Es sind also jeweils mehrere Faktoren bei einer Beurteilung der Übernahme von Architektur- und Dekorationsformen durch die Italiker zu berücksichtigen und deren unterschiedliche Bedeutung in Betracht zu ziehen.

So ist die Wahl poseidoniatischer Architekturformen in der Siedlung am Monte Pruno vor allem als Folge der guten Kenntnis der Großbauten im nahe gelegenen Poseidonia und der Verfügbarkeit dort geschulter Handwerker zu sehen. Auch in Pompeji beauftragte man mit der Verzierung des ersten größeren Kultbaus eine in der Nähe ansässige kampanische, genauer: cumanische Werkstatt. Daß die Arbeit dieser Meister darüber hinaus dem ästhetischen Empfinden einer breiten Mehrheit der ansässigen Bevölkerung entsprach, ist aufgrund des öffentlichen Charakters der Gebäude vorauszusetzen. Der Einfluß der neben diesen praktischen und ästhetischen Kriterien wirksamen politisch-ideologischen Faktoren ist allerdings nicht mehr nachvollziehbar. Das Aussehen dieser öffentlichen Bauten allein erlaubt daher keine weiter reichenden Schlüsse auf Art und Ausmaß der politischen Beziehungen der italischen Siedlungsgemeinschaften zu den Herkunftsorten der für sie tätigen Handwerker.

Eine stärkere Aussagekraft hinsichtlich politischer Kontakte hat der Nachweis der Übernahme neuer, bis dahin nicht verwendeter Formen und Stilrichtungen in Siedlungen, die bereits eine fest verwurzelte Tradition monumentaler Architektur hatten. Eine solche Neuerung bedeutete der Bau des dorischen Tempels am Forum Triangulare in Pompeji sowie die Errichtung von Kultbauten im etruskisch-griechischen Stil Nordkampaniens im südlichen Stadtheiligtum des griechischen Poseidonia. Dabei standen wohl kaum praktische oder technische Gesichtspunkte im Vordergrund, da unter diesen ein Auftrag an eine der ansässigen bzw. eine der bereits bekannten Werkstätten vergeben worden wäre. Die für die Errichtung dieser Großbauten Verantwortlichen nahmen vielmehr Kontakt mit Handwerkern auf, die bis dahin nur in weit entfernt gelegenen Siedlungen tätig gewesen waren. Dies spricht dafür, daß es bereits im Vorfeld dieser Baumaßnahmen auch zu politischen Kontakten dieser Siedlungen - im konkreten Fall Pompeji und Poseidonia - kam, und die Wahl des neuen Dekorationsstils Ausdruck dieser politischen Zusammenarbeit war. Darüber hinaus spricht die Akzeptanz der neuartigen Formen durch die jeweils ansässige Bevölkerung für regelmäßige wechselseitige Besuche und wirtschaftliche Kontakte durch Händler und Handwerker beider Siedlungen.

Ähnliches gilt wohl für Fratte, wo im späten 6 . und dann vor allem in der ersten Hälfte des 5. Jh.s neben den bis dahin üblichen nordkampanischen Architekturformen und Kunstwerken zunehmend poseidoniatische Formen und Erzeugnisse verwendet wurden. In Fratte kann das Zusammenleben von Italikern, Etruskern und poseidoniatischen Griechen sogar direkt nachgewiesen werden $^{134}$. Neben zahlreichen Belegen einer Verwendung des achäischen Alphabets durch hier ansässige Italiker und Etrusker gibt vor allem die Aufschrift einer im frühen 5. Jh. in Poseidonia angefertigten Olpe deutlich Aus-

133 Die unterschiedlichen zur Wahl einer bestimmten Form des Baus und seiner Dekoration verantwortlichen Faktoren und ihr Zusammenspiel wurde jüngst vor allem von D. Mertens, 1994, s. o. Anm. 6, 196-207, ausführlich diskutiert.

134 G. Colonna, Le iscrizioni etrusche di Fratte, in: Fratte, 1990, 301-309; vgl. auch den im selben Band publizierten Beitrag von L. Cerchiai, Fratte e Pontecagnano, 310313. 
kunft über die intimen Beziehungen von Angehörigen aller dreier Volksgruppen: auf diesem Gefäß sind Griechen, Etrusker und Italiker als Partner beim Liebesspiel genannt ${ }^{135}$. Auch andere Inschriften aus Fratte zeigen, daß sich hier Poseidoniaten über einen längeren Zeitraum aufhielten und auch Italiker des griechischen Alphabets von Poseidonia bedienten ${ }^{136}$. Den Nachweis einer Übernahme des poseidoniatischen Alphabets durch eine Etruskerin erbringt die Besitzerinschrift einer gewissen Letia auf dem Griff eines Bronzespiegels, dessen Auffindung in Fratte allerdings nicht restlos gesichert ist ${ }^{137}$.

\section{ZUSAMMENFASSUNG}

Bei ihrer Landnahme in der Küstenebene südlich des Sele ging es den Griechen vor allem um den dauerhaften Besitz größerer wirtschaftlich nutzbarer Flächen. Daher nahmen die Neusiedler wohl schon bald die gesamte Fruchtebene in Besitz. Dies dürfte zu einer Abwanderung zumindest eines bedeutenden Teils der vormals ansässigen italischen Bauern geführt haben, die sich nun wohl im östlichen Bergland niederließen. Das bereits im frühen 6. Jh. in Fonte, am Übergang zum hügelig-gebirgigen Hinterland eingerichtete Heiligtum der Hera dürfte daher von den Griechen als ein Grenzheiligtum gegenüber den Italikern gegründet worden sein. Der Kult der Stadtgöttin sollte hier wohl die politischen Interessen der poseidoniatischen Bauern vertreten, die bereits im frühen 6. Jh. den Besitz der gesamten Fruchtebene für sich beanspruchten, auch wenn sie zunächst nur zu land- bzw. forstwirtschaftlicher Arbeit in das Hinterland ihrer Siedlung kamen und dort erst im 4. Jh. in größerer Zahl seßhaft wurden.

Das an der Mündung des Sele gegründete Heraion sollte zwar wohl ebenfalls die Funktion eines Grenzheiligtums erfüllten, diente also einer Bestätigung der natürlichen Begrenzung des von den Griechen beanspruchten Territoriums im Norden, gleichzeitig aber sollte dieses Heiligtum der Stadtgöttin die Bereitschaft der Griechen zu einem friedlichen Zusammenleben mit ihren Nachbarn jenseits des Flusses zum Ausdruck zu bringen. Daher wurde das Heraion wohl von Anfang an als ein Kultort eingerichtet, der grundsätzlich auch den Etruskern und Italikern offen stand.

Handelsinteressen dürften der entscheidende Faktor dieses Strebens nach friedlichen Verhältnissen gewesen sein und ganz allgemein den politischen Kontakt von Italikern, Etruskern und Griechen in Südkampanien entscheidend gefördert haben. Treibende Kraft zur Gründung der Handelsnie-

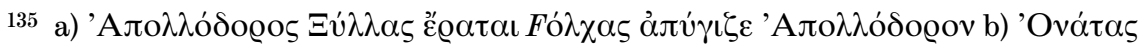

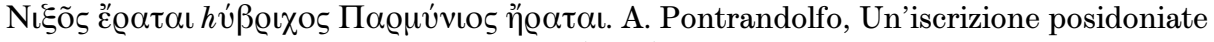
in una tomba di Fratte di Salerno, AION 9 (1987) 55-63, figg. 20-22; SEG 37,817; R. Arena, 1996 s. o. Anm. 23, 58f. Nr. 33 mit Umzeichnung. Die genannten Apollodor und Onatas sind sicher Griechen, Vulca ein Etrusker. Die übrigen Namen Xylla, Nixos, Hybrichos und Parmynon sind bisher nicht belegt, am ehesten aber als italische Namen in griechischer Schreibung zu deuten (A. Pontrandolfo, 1987, 59f. 61).

${ }^{136}$ Als Besitz des Poseidoniaten Dymeiadas $(\Delta v \mu \varepsilon ı \alpha ́ \delta \alpha)$ ist eine Bronzeolpe aus Fratte ausgewiesen; IG XIV, 694; LSAG $^{2}$, 252. 260 Nr. 6; R. Arena, 1996 s. o. Anm. 23, 56 Nr. 31. Trebis, der sich in der ersten Hälfte des 5. Jh.s als Besitzer auf einer attischen Schale verewigte, war mit Sicherheit ein Osker, im Fall des Visuvos bzw. Isyllos wurde der Name je nach Lesung als italisch (FıvvFos; G. Colonna, 1994, s. o. Anm. 102, 359 Anm. 78; ders. in: Fratte, 1990, 306) oder griechisch (Fıv́̉os; R. Arena, 1996, s. o. Anm. 23, 54 Nr. 29) gedeutet.

137 G. Colonna, in: Fratte, 1990, 307, Fig. 523. 
derlassungen am Kap Agropoli und an der Mündung des Sele war wohl die poseidoniatische Mutterstadt Sybaris, deren Händler hier Handel mit dem etruskischen Pontecagnano betreiben konnten und Stationen auf der Handelsroute weiter nach Norden, nach Kampanien und in das etruskische Kernland, vorfanden.

Handelsinteressen der griechischen wie etruskischen Siedlungsgemeinschaften Südkampaniens führten wohl auch dazu, daß die praktischen Voraussetzungen wirtschaftlicher Aktivitäten geschaffen wurden, also das Bereitstellen öffentlicher Flächen als Hafen-, Markt- und Stapelplätze sowie die Errichtung entsprechender öffentlicher Großbauten (Säulenhallen, schützende Mauern). Eine weitere öffentliche Maßnahme war die Einrichtung von Kultstätten am Ort des Warenaustausches, wobei die Angehörigen der drei unterschiedlichen Volksgruppen dieselbe Gottheit nach jeweils eigener Tradition verehrten. Diese Götter - Apollo in Pontecagnano und wohl auch Pompeji sowie Hera an der Selemündung - dürften die rechtlichen Grundlagen des wirtschaftlichen Kontaktes zum Ausdruck gebracht haben und vertraten damit formal die lokale politische Autorität, die von den italischen, etruskischen und griechischen Handelspartnern gleichermaßen anerkannt wurde.

Auf einer allgemeineren Ebene weist auch die Übernahme von Bauformen und Stilelementen zur Gestaltung öffentlicher Großbauten Beziehungen der unterschiedlichen Volksgruppen nach. Da allerdings dabei für die italischen Siedlungsgemeinschaften Südkampaniens, die im 6. Jh. noch auf keine eigene Tradition monumentaler Steinarchitektur zurückgreifen konnten, praktische und funktionale Fragen ebenso eine Rolle spielten wie ästhetisches Empfinden und politisch-ideologische Vorstellungen, sind weitreichende Schlüsse auf politische Beziehungen der Italikern zu Etruskern und Griechen ausschließlich auf Basis dieser Evidenz nicht zulässig.

Anders zu beurteilen ist der Austausch von Bauformen und Dekorationsstilen zwischen Pompeji und Poseidonia. Dabei wurden in beiden Siedlungen bewußt und ohne zwingende praktische Notwendigkeit neue Formen in ein bereits etabliertes System der Gestaltung öffentlicher Großbauten aufgenommen: in Pompeji die griechisch-poseidoniatische Steinarchitektur (Tempel am Forum Triangulare), in Poseidonia die Verzierung von Tondächern in kampanischer Tradition (Gebäude im südlichen Stadtheiligtum). Dies spricht dafür, daß diese Neuerungen bewußt erfolgten, um die engen Beziehungen und die Zusammenarbeit beider Siedlungsgemeinschaften auch auf politischer Ebene zum Ausdruck zu bringen. Ähnliches ist für die italische Siedlung in Fratte wahrscheinlich und wohl auch für jene am Monte Pruno anzunehmen. Letztere dürfte daher wohl in Folge einer deutlich intensivierten Nutzung der Handelsroute von Poseidonia in das Vallo di Diano und weiter nach Sybaris und Metapont gegründet worden sein; solches ist am ehesten vor dem Hintergrund der im Zuge der Zerstörung von Siris verstärkten politischen Spannungen zwischen Rhegion und den achäischen Städten Sybaris, Metapont und Kroton zu sehen ${ }^{138}$, die zu Problemen bei der Durchfahrt achäischer Schiffe durch die Meerenge von Messina geführt haben könnte.

Der Hintergrund der politischen Kontakte Poseidonias mit Pompeji im späten 6. und frühen 5. Jh. ist nicht direkt bezeugt, sie sind aber wohl ebenfalls auf jene Faktoren zurückzuführen, die die Außenpolitik dieser Siedlungen in diesem Zeitraum entscheidend beeinflußten. Für Pompeji spielte damals der politische Einfluß von Cumae im Golf von Neapel eine entscheidende

${ }^{138} \mathrm{Zu}$ diesen Spannungen bereits oben Anm. 25. 
Rolle ${ }^{139}$, während sich die Poseidoniaten seit der Gründung von Velia an der Südgrenze ihres Territoriums mit den Phokäern als neue Nachbarn und Handelskonkurrenten auseinandersetzen mußten ${ }^{140}$. Von einschneidender Bedeutung für Poseidonia war weiters die Zerstörung ihrer Mutterstadt Sybaris im Jahr 510 ${ }^{141}$, was wohl auch zum Verlust eines bedeutenden Absatzmarktes im Süden führte und die Poseidoniaten dazu gezwungen haben dürfte, sich nach neuen Handelspartnern umzusehen.

Vorgelegt von k. M. Luciana Aigner-Foresti

in der Sitzung am 6. Oktober 2000.

139 Die oben schon erwähnte Errichtung des Mauerrings um Pompeji und dessen Zerstörung im frühen 5. Jh. ist deutlicher Hinweis auf eine militärische Bedrohung im späteren 6. und frühen 5. Jh. Diese wurde schon von St. de Caro, 1985, s. o. Anm. 90, 112 f., mit der Ausbreitung von Cumae im Golf von Neapel verbunden. Weiterführende historische Überlegungen in diesem Sinn vor allem bei M. Cristofani, La fase „etrusca“ di Pompei, in: Pompei, Hg. F. Zevi, Neapel 1992, 7-20, und mit starkem Bezug zum archäologischen Befund F. Zevi, 1998, s. o. Anm. 90, 3 ff. Allgemein zur politischen Bedeutung Cumaes im späten 6. und frühen 5. Jh. Cerchiai, 1995, 150-153; E. M. de Juliis, Magna Grecia. L'Italia meridionale dalle origini leggendarie alla conquista romana, Bari 1996, 137-139; zur damit verbundenen politischen Propaganda N. Valenza Mele, Eracle euboico a Cuma - La Gigantomachia e la Via Heraclea, in: Recherches sur les cultes grecs et l'Occident I, Neapel 1979 (Cahiers du Centre J. Berard V) 19-51. Zu den archäologischen Belegen einer cumanischen Ausdehnung im Golf von Neapel (Misenum, Dikaiarcheia, Palaiopolis) E. Greco, Archeologia della Magna Grecia, Rom/Bari 1992, 18f.

140 Die Gründung von Velia ist ausführlich bei Hdt. 1,163-167 beschrieben, wobei

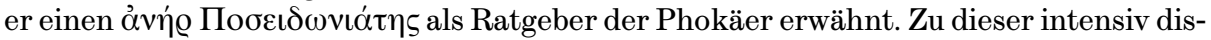
kutierten Passage aus poseidoniatischer Sicht G. P. Carratelli, Per la storia di Poseidonia, in: Poseidonia-Paestum, 26-28, sowie mit weiteren Literaturangaben E. Greco, 1992 , s. o. Anm. 139, 80 ff. und E. de Juliis, 1996, s. o. Anm. 139, 171 ff. Bewaffnete Auseinandersetzungen zwischen Poseidonia und Velia sind bei Strab. 6,1,1 (C 252) genannt. Zur handelspolitischen Bedeutung Velias im späteren 6. und 5. Jh., die ein plausibler Anlaß derartiger Kämpfe war, zuletzt F. di Bello, 1997, s. o. Anm. 57, bes. 47 f.

141 Die Zerstörung der blühenden achäischen Stadt Sybaris war in mehrfacher Hinsicht ein traumatisches Ereignis, nicht nur nur für die Bewohner Süditaliens, sondern auch für das griechische Mutterland (besonders deutlich bei Hdt. 6,21). Die vielfältigen historischen Aspekte dieses auch von Diod. 12,9,3-6 geschilderten und 11,90,3 in das Jahr 510 datierten Ereignisses wurden zuletzt vor allem von M. Lombardo, Da Sibari a Thurii, in: Sibari e la Sibaritide, Atti del XXXII. Conv. di studi sulla Magna Grecia 1992, Tarent 1993, 255-298, behandelt; ausführliche Hinweise auf die wichtigsten früheren Arbeiten zu diesem Thema bei G. de Sensi Sestito, La Calabria in età arcaica e classica, in: Storia della Calabria antica, Hg. S. Settis, Reggio di Calabria/Rom 1988, 297.

Daß sich ein Teil der Sybariten nach der Zerstörung ihrer Stadt auch in Poseidonia niedergelassen und hier verstärkten Einfluß auf das politische Geschehen genommen haben könnte, erscheint plausibel, auch wenn Hdt. 6,21 als Zufluchtsorte der Sybariten nur Laos und Skidros erwähnt. Allerdings sind die dafür angeführten archäologischen und numismatischen Hinweise aus Poseidonia selbst (Errichtung eines Hypogäum auf der Agora, Prägung von Münzen mit der Aufschrift Viis, womit vielleicht der Gründer von Sybaris, Is von Helike, gemeint sein könnte; dazu etwa G. P. Carratelli, 1987, s. o. Anm. 140, $28 \mathrm{ff}$.) aufgrund der unsicheren Datierung mit Vorsicht zu interpretieren, wie jüngst M. Lombardo, Atti del XXXII. Conv. di Studi sulla Magna Grecia 1992, 274, sowie A. Mele, Storia di Poseidonia tra VI e V secolo a. C., in: I Greci in Occidente: I Greci in Occidente. Poseidonia e i Lucani, Hgg. M. Cipriani/F. Longo, Neapel 1996, 18, betonten. 